\title{
INFLUENCE D'UN ÉCRAN ÉLECTRIQUE EN AVAL D'UNE USINE HYDROÉLECTRIQUE SUR LE COMPORTEMENT DE REMONTÉE DU SAUMON ATLANTIQUE (SALMO SALAR)
}

\author{
C. Gosset (1), F. TRAVAde (2), C. GARAICoEChEA (1)
}

(1) Station d'Hydrobiologie, INRA, BP 3, 64310 Saint-Pée-sur-Nivelle, France

(2) Electricité de France, Direction des Etudes et Recherches, 6 Quai Watier, 74800 CHATOU, France.

\section{RÉSUMÉ}

Le comportement de remontée de sept saumons atlantiques a été observé en aval d'un écran électrique répulsif implanté dans le canal de fuite d'une usine hydroélectrique située sur la Nive à $20 \mathrm{~km}$ de la mer.

Le suivi des saumons par la technique du radiopistage montre qu'ils ne restent pas bloqués en position d'attente devant l'écran et ne dévalent pas d'une façon importante après avoir subi l'effet du champ électrique. Bien qu'il existe une individualité très marquée dans les mouvements migratoires, il n'a pas été constaté de différence sensible entre le comportement des saumons repoussés par l'écran et ceux qui ont vagabondé dans la basse Nive sans avoir pénétré dans la zone d'action de l'écran. L'efficacité répulsive de l'écran a été confirmée.

Par ailleurs, on a observé un ralentissement important de l'activité des saumons dans la basse Nive pendant la période estivale et des remontées rapides vers les zones de frayères lors des coups d'eau d'automne.

\section{INFLUENCE OF AN ELECTRIC SCREEN DOWNSTREAM FROM AN HYDROELECTRIC PLANT ON UPSTREAM MIGRATION OF THE ATLANTIC SALMON (SALMO SALAR)}

\section{SUMMARY}

Upstream migration behaviour of seven Atlantic salmon was observed downstream from a repelling electric screen set in an hydroelectric plant channel discharge on the Nive River, $20 \mathrm{~km}$ from the sea.

Results of radio-tracking the fish show that they do not remain in a waiting position in front of the screen and do not move far downstream after the electric field effect. Diverse migration movements were observed. However, no difference in behaviour was noted between salmon which were in contact with the screen and those which were not, and stayed in the lower part of the River Nive. Repelling efficiency of the screen was confirmed. 
Salmon activity was greatly decreased in the lower Nive River during the summer period, and quick upstream movements to spawning zones during autumn peak flow rates was also observed.

\section{INTRODUCTION}

Lors de leur migration vers l'amont, les saumons empruntent fréquemment les canaux de fuite des microcentrales hydroélectriques dont le. débit est très souvent supérieur à celui de la rivière. Pour éviter que ces poissons ne restent bloqués devant la sortie des turbines pendant une période suffisamment longue pour compromettre la fraie, les exploitants de microcentrales sont conduits à construire, au niveau de l'usine, des passes ou échelles à poissons qui permettent le libre accès au canal d'amenée. Cependant, ces passes ne sont pas toujours techniquement réalisables ou représentent, dans la plupart des cas, un investissement financier important. De ce fait, les exploitants sont de plus en plus tentés par l'installation d'un écran électrique de remontée. Cet écran, installé au confluent de la rivière et du canal de fuite est destiné à empêcher les saumons de pénétrer dans le canal de fuite et à les inciter à poursuivre leur remontée par le cours principal. Utilisé comme ouvrage complémentaire d'une passe aménagée dans le barrage principal de la rivière - cette passe est toujours plus rudimentaire et donc beaucoup moins coûteuse que celle qui est située à l'usine hydroélectrique - il peut donc, soit constituer la seule solution pour faciliter la migration d'amontaison lorsqu'il n'est pas possible de construire une passe à l'usine hydroélectrique, soit représenter, dans certains cas, une alternative moins onéreuse à la construction de cette passe.

La solution écran semble a priori et à bien des égards (coût, rapidité d'installation, entretien) séduisante. Cependant, si le rôle de barrière quasi-infranchissable de l'écran de remontée a été maintes fois démontré (HARTLEY et SIMPSON, 1968 ; STEWART, 1990), il n'existe pas, à notre connaissance, d'étude concernant son influence sur les déplacements consécutifs à la répulsion ou, d'une façon plus générale, concernant son impact sur le comportement migratoire. En effet, les observations faites in situau Royaume-Uni essentiellement (HARTLEY et SIMPSON, 1968) ont été le plus souvent visuelles et effectuées dans une zone voisine de l'écran, sans suivi de longue durée des poissons. Depuis 1968, cette catégorie d'écrans ne semble pas avoir fait l'objet d'études (STEWART, 1990), si ce n'est un travail plus spécifique de JOHNSON (JOHNSON et al., 1990) concernant le guidage des saumons de remontée vers une station de piégeage.

Par ailleurs, des observations récentes (BOMASSI et BRUGEL, 1991) ont montré que des saumons (de printemps et du début de l'été essentiellement) arrêtés par une microcentrale n'ont pas séjourné dans le canal de fuite. Ils ont effectué régulièrement des replis vers l'aval et sont retournés dans ce même canal tant que les conditions environnementales n'ont pas été modifiées, sans prospecter d'autres voies de passage. Ces poissons ne se sont engagés dans le bras court-circuité que dans des conditions particulières : arrêt prolongé de l'usine en période nocturne, conjugué de préférence à une augmentation de débit. Ompouvait se demander si un comportement similaire serait observé sur un autre site et surtout si ce comportement était susceptible d'être modifié par la mise en service d'un écran électrique. En effet, il apparaît clairement que si les saumons repoussés par un écran de remontée demeurent en attente en aval de celui-ci sans poursuivre leur migration par le bras court-circuité, ce dispositif perd en grande partie son intérêt.

Nous avons pensé qu'un suivi comportemental effectué par radiopistage, moyen bien adapté à ce type de surveillance, permettrait de répondre à ces deux questions. En effet, cette technique moderne permet de suivre de façon précise les déplacements des animaux repoussés parl'écran électrique à l'intérieur d'une zone expérimentale environnant l'écran, mais suffisamment étendue pour permettre des observations significatives.

II semble évident a priori que l'emplacement idéal pour l'implantation d'un écran se situe, comme mentionné ci-dessus, au confluent entre le canal de fuite et la rivière, de façon à éviter un éventuel blocage des poissons dans le canal. Cette solution n'a pu, pour l'instant et pour différentes raisons (moyens financiers insuffisants, en particulier), être retenue. Par contre, un écran électrique expérimental déjà installé devant la sortie des turbines d'une microcentrale (Photo 1), en amont d'un canal de fuite de $600 \mathrm{~m}$ de longueur environ, offrait une opportunité intéressante pour effectuer un tel travail. On peut en effet penser que si les saumons ne restent pas en attente devant l'écran dans un cas très défavorable (retour en arrière important), 
la libre circulation sera a fortiori mieux assurée si l'écran est implanté à l'entrée du canal de fuite.

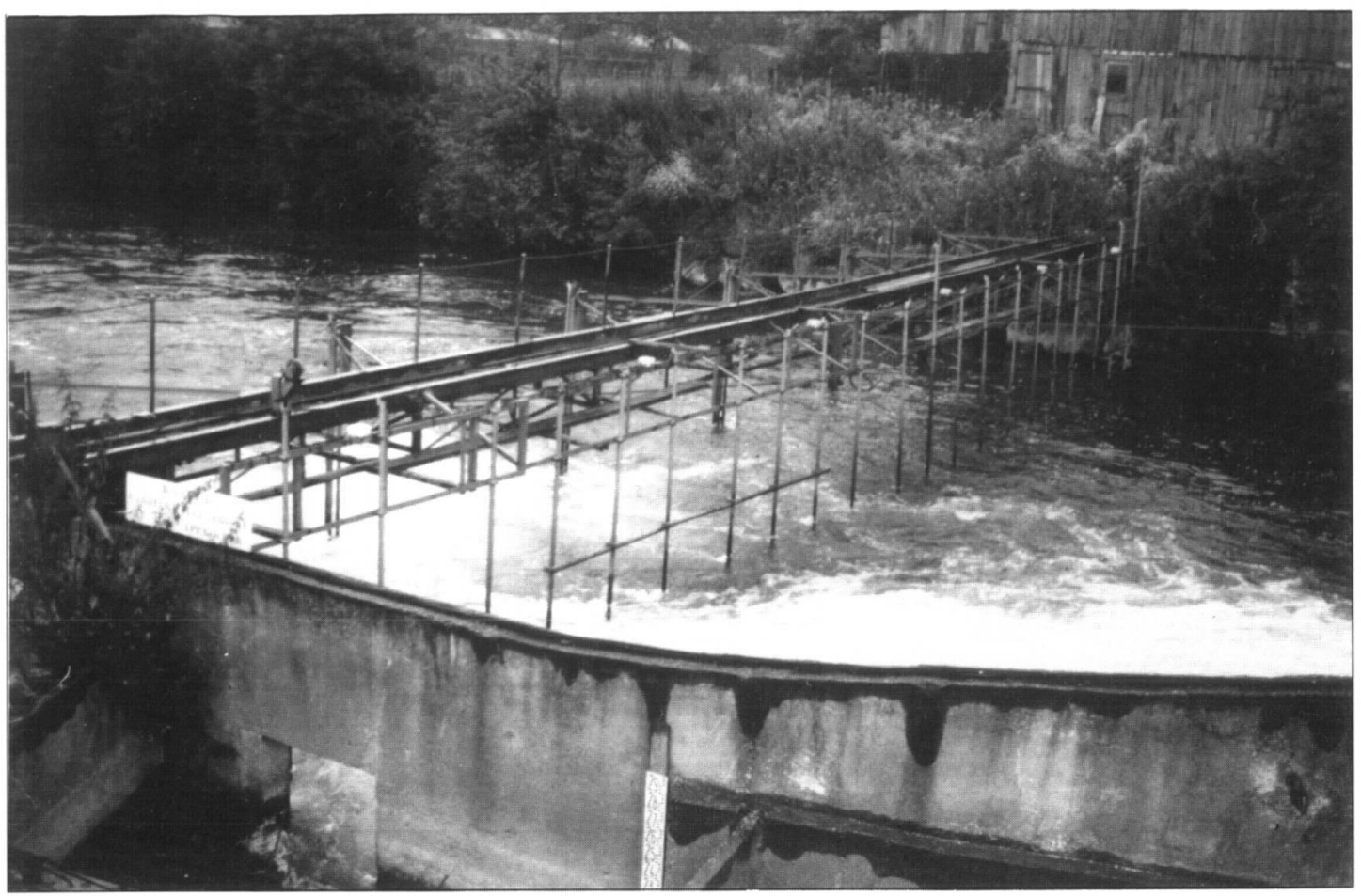

Photo 1 : Vue de l'écran électrique et de l'entrée de la passe à poissons de Chopolo.

Photo 1 : View of the Chopolo electric screen and fish pass entrance.

\section{MATÉRIEL ET MÉTHODES}

\subsection{Description du milieu et du matériel}

La zone d'expérimentation choisie se situe sur la Nive, affluent de l'Adour, à hauteur d'Ustaritz soit à environ $20 \mathrm{~km}$ de la mer (Fig. 1). Le module interannuel de la Nive dans cette zone est de $35,5 \mathrm{~m}^{3} \cdot \mathrm{s}^{-1}$ et en 1991 le débit moyen de cette rivière a été de $23 \mathrm{~m}^{3} \cdot \mathrm{s}^{-1}$. Le débit journalier est très variable en fonction des conditions météorologiques (de $6 \mathrm{~m}^{3} . \mathrm{s}^{-1}$ en étiage à plus de $300 \mathrm{~m}^{3} \cdot \mathrm{s}^{-1}$ en cas de coups d'eau importants).

Cette zone (Fig. 2) est délimitée par 3 obstacles à la migration. En aval le barrage de la Lyonnaise des Eaux, en amont le barrage du Bourg et la centrale hydroélectrique de Chopolo. Dans ce système hydraulique relativement complexe, on trouve un certain nombre de points et sites caractéristiques:

- le barrage de la Lyonnaise des Eaux : ouvrage rustique délimitant l'influence des marées (exception faite des grandes marées qui recouvrent le barrage) franchissable, à marée haute, même par débit relativement faible ; de 3 à $4 \mathrm{~m}$;

- la gravière : plan d'eau créé par l'extraction de graviers d'une profondeur moyenne

- la centrale d'Arki : microcentrale hydroélectrique en exploitation ; puissance maximale : $250 \mathrm{Kw}$; débit turbiné : 4 à $14 \mathrm{~m}^{3} \cdot \mathrm{s}^{-1}$ en fonction du débit de la Nive ; à poissons ;

- le barrage d'Arki : ouvrage important de $2 \mathrm{~m}$ de hauteur environ, équipé d'une passe 


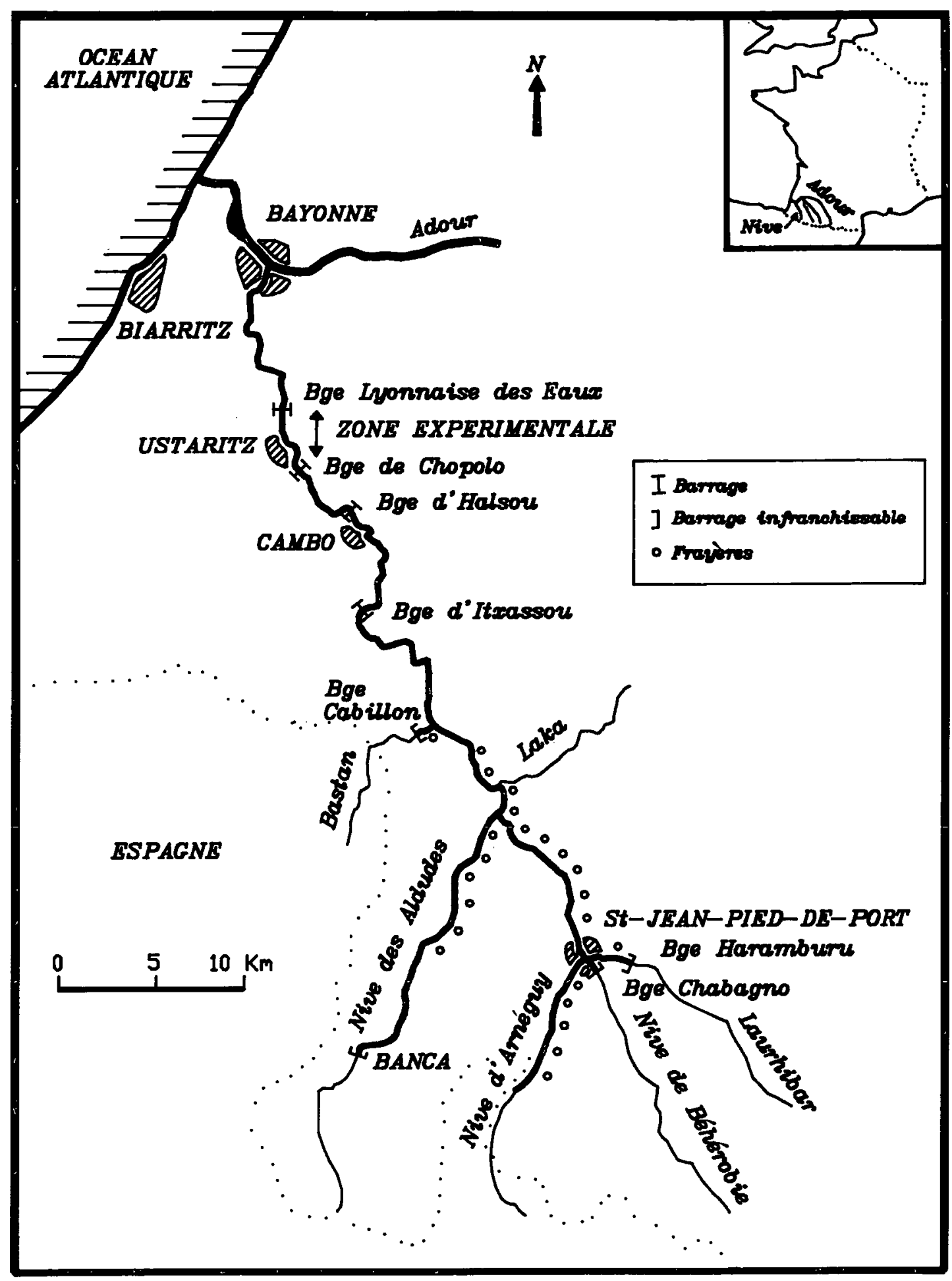

Figure 1 : Bassin de la Nive.

Figure 1 : Water course of the river Nive. 


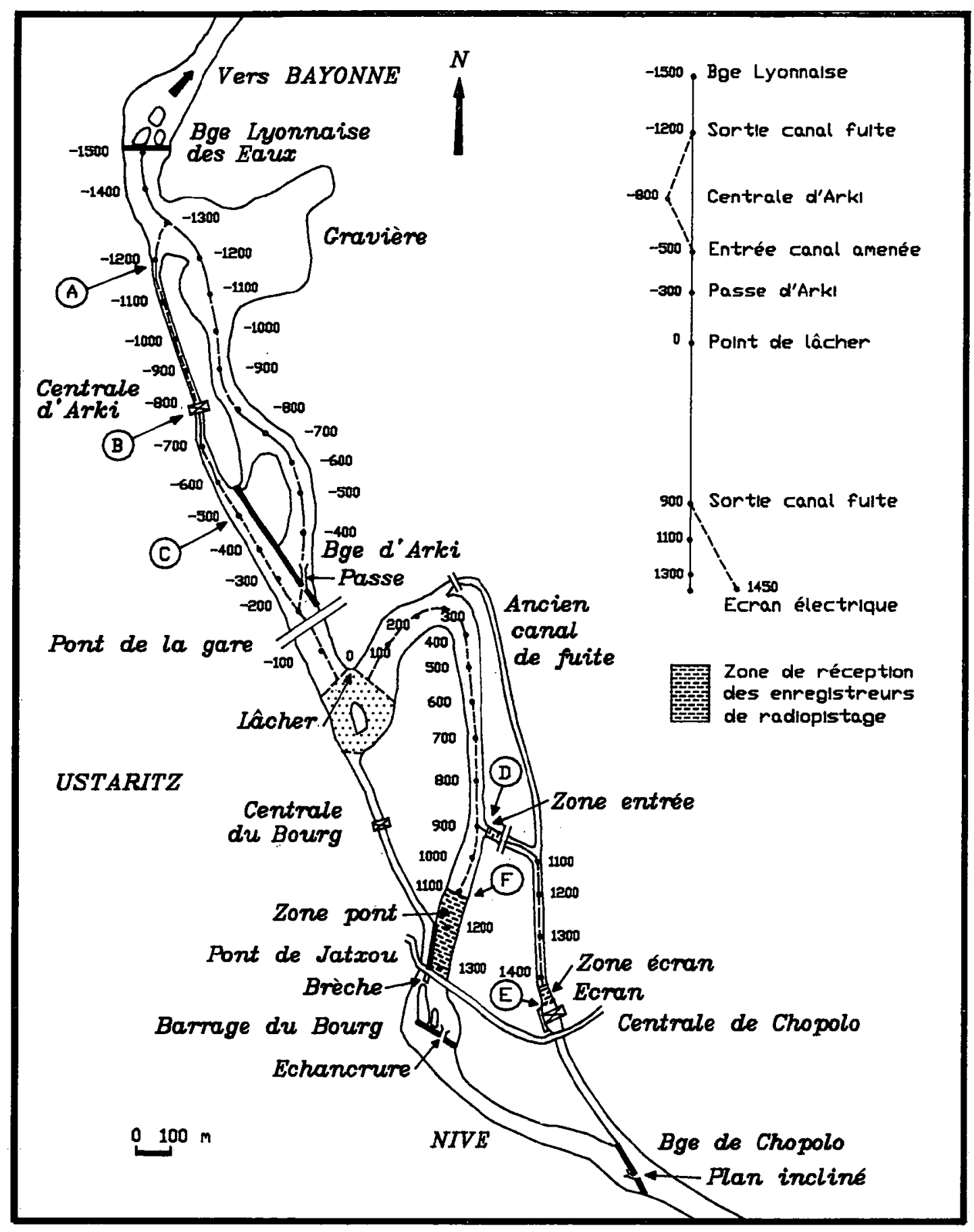

Figure 2 : Zone expérimentale.

Figure 2 : Experimental zone. 
- la centrale du Bourg : microcentrale hydroélectrique à l'arrêt pendant la durée de l'expérimentation. Début 91, une crue a provoqué la formation d'une brèche dans la digue séparant le canal d'amenée de la Nive, à hauteur du pont de Jatxou. Ceci a entraîné non seulement la mise à sec de ce canal mais également la création d'un passage pour les poissons, pendant la plus grande partie de l'année ;

- le barrage du Bourg : ouvrage de faible hauteur équipé d'une échancrure maintenant un débit minimum de 0,6 à $1 \mathrm{~m}^{3} \cdot \mathrm{s}^{-1}$;

- le barrage de Chopolo : ouvrage rustique de 1,5 $\mathrm{m}$ de hauteur environ, équipé d'un plan incliné pour le passage des migrateurs ;

- l'ancien canal de fuite de Chopolo : canal court-circuité par le bras réaménagé se jetant dans la Nive au niveau de la "zone entrée" mais dans lequel subsiste un débit de l'ordre de 0,2 à $0,5 \mathrm{~m}^{3} \cdot \mathrm{s}^{-1}$;

- la centrale de Chopolo : microcentrale hydroélectrique en exploitation ; puissance maximale : $300 \mathrm{~kW}$ : débit turbiné : 4 à $16 \mathrm{~m}^{3} \cdot \mathrm{s}^{-1}$. Cette centrale est équipée d'une passe à bassins successifs dans laquelle est installée une chambre de capture. Immédiatement en aval de l'entrée de la passe et à environ $15 \mathrm{~m}$ de la sortie des turbines, est installé l'écran électrique de remontée (Fig. 3). Il a été édifié à cet endroit dans un but expérimental afin de pouvoir compter aisément les poissons l'ayant franchi.

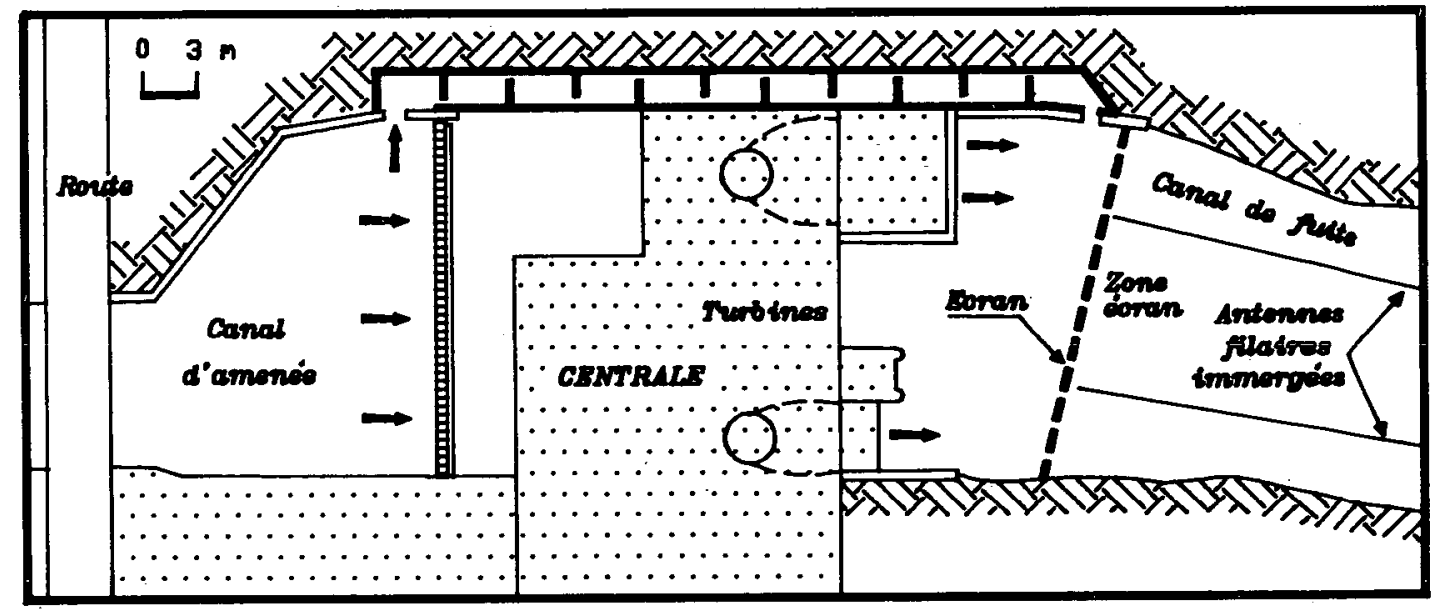

Figure 3 : Schéma du site de la centrale hydroélectrique de Chopolo.

Figure 3 : Diagram of the Chopolo hydroelectric plant site.

L'écran électrique de remontée faisant l'objet de l'étude fonctionne depuis 1989 et a été conçu pour effectuer des mesures d'efficacité en fonction des caractéristiques mécaniques (nombre de rangées, écartement des barreaux...) et électriques (types de courant, voltage...) aisément modifiables.

Ce dispositif de $22 \mathrm{~m}$ de longueur est constitué de 2 rangées de barreaux cylindriques verticaux espacées de $3 \mathrm{~m}$. Lorsque les 2 rangées d'électrodes sont alimentées la rangée aval fait office d'anode, la rangée amont représente la cathode.

Dans le cadre de cette étude, l'écran a fonctionné suivant le modèle de type ERZU-1 (STRAKHOV, 1965), c'est-à-dire en utilisant une seule rangée scindée en sections de 8 électrodes. L'alimentation est du type : salves d'impulsions non polarisées - fréquence des salves : $2 \mathrm{H}_{\mathrm{z}}$; durée des salves : $40 \mathrm{~ms}$. Le taux de répulsion obtenu avec cet écran, mesuré sur des salmonidés d'une longueur supérieure à $50 \mathrm{~cm}$, est de $100 \%$ (GOSSET, 1989). 
Le suivi a étémené à l'aide de matériels d'origine américaine de marque A.T.S. (Advanced Telemetry System), soit :

- récepteurs type "Challenger Model $2000 \mathrm{~B}$ " à balayage (fréquence de balayage : 2 secondes à $16 \mathrm{mn}$ ) et programmables (2000 canaux entre 48 et $49,990 \mathrm{MH}_{\mathrm{z}}$ );

- enregistreur type "Easterline Angus" (244 canaux) sur papier, possédant une autonomie de 10 jours environ avec une vitesse de déroulement de $7,6 \mathrm{~cm}$ par heure. Ces enregistreurs sont couplables aux récepteurs "Challenger" par l'intermédiaire d'interfaces séparées ;

- antennes boucle $(40 \times 40 \mathrm{~cm})$ directionnelles ;

— antennes filaires dont la longueur est adaptée à la longueur de la zone à surveiller ;

- émetteurs "Model 5 " (diamètre : $19 \mathrm{~mm}$; longueur : $66 \mathrm{~mm}$; poids : $25 \mathrm{~g}$; autonomie minimale : 200 jours) avec option "circuit de mortalité" (la fréquence de modulation double si l'émetteur reste immobile plus de $24 \mathrm{~h}$ ) — plage des fréquences utilisées : 48,410 $\mathrm{MH}_{\mathrm{z}}$ à $48,530 \mathrm{MH}_{z}$.

\subsection{Méthode expérimentale}

Le 10 juin 1991, 8 saumons ont été capturés au piège de la passe à poissons de Chopolo. Ces poissons étaient tous argentés et donc très récemment remontés de la mer. Ils n'ont très vraisemblablement pas été exposés au courant électrique de l'écran, à l'arrêt depuis plusieurs jours.

L'un deux qui présentait une blessure importante à la bouche a été relâché, les 7 autres ont été immédiatement anesthésiés (phénoxy 2 éthanol) puis mesurés et ont subi un prélèvement d'écailles. Les principales caractéristiques de ces saumons sont données au tableau I. Ils ont été ensuite marqués par insertion stomacale d'une marque radio préalablement lubrifiée à la glycérine. Cette méthode de marquage a été décrite par différents auteurs (MC LEAVE et al., 1978 ; BURGER, 1981 ; SOLOMON, 1982 ; BARIL et GUENEAU, 1986 ; STEINBACH et al., 1986 ; TRAVADE et al., 1989).). L'antenne souple de $30 \mathrm{~cm}$ de longueur a été insérée en arrière de l'arc branchial postérieur et laissée libre le long du corps.

Ces saumons marqués ont été transportés par un véhicule équipé d'une cuve et relâchés environ 20 minutes plus tard, dans la Nive, à $1.500 \mathrm{~m}$ en aval (cote zéro) dans une zone à courant très faible.

Deux méthodes classiques de radiopistage (MC LEAVE et al., 1978; BURGER et al, 1981 ; SOLOMON, 1982 ; BARIL et GUENEAU, 1986 ; TRAVADE et al., 1989) ont été utilisées :

— un repérage manuel à l'aide d'une antenne boucle. L'opérateur se déplace à pied le long des berges ou en voiture, auquel cas il effectue son repérage à partir de points "stratégiques" situés à différents endroits de la zone d'expérimentation. Ces points ont été choisis principalement en fonction de leur accessibilité et de leur situation favorable pour un repérage précis par goniométrie (les 2 ou 3 directions nécessaires étant déterminées par l'orientation de l'antenne à l'extinction du signal) ;

- un repérage automatique par enregistrement du signal reçu. Ce repérage est effectué au moyen de 3 ensembles récepteurs - interface - enregistreurs qui assurent une surveillance permanente à l'intérieur de 3 zones importantes (Fig. 2) :

- une zone dite "zone écran" située à l'aval immédiat de l'écran de 20 à 25 m de large et de $30 \mathrm{~m}$ de longueur. Cette zone est "couverte" par 2 antennes filaires immergées de même longueur (Fig. 3). 
Tableau I : Caractéristiques des saumons radio-marqués.

Table I : Characteristics of the radio-tagged salmons.

\begin{tabular}{ccccc}
\hline $\begin{array}{c}N^{\circ} \text { des } \\
\text { saumons }\end{array}$ & $\begin{array}{c}\text { Longueur } \\
(\mathrm{cm})\end{array}$ & $\begin{array}{c}\text { Poids } \\
(\mathrm{g})\end{array}$ & Sexe & Age \\
\hline 1 & 70 & 2630 & $\mathrm{M}(1)$ & $\begin{array}{c}\text { Ecailles } \\
\text { régénérées }\end{array}$ \\
2 & 81,5 & 4750 & $\mathrm{~F}(?)$ & $1.2^{+}$ \\
3 & 79,5 & 5520 & $\mathrm{~F}(?)$ & $1.2^{+}$ \\
4 & 63 & 2230 & $\mathrm{M}(1)$ & $1.1^{+}$ \\
5 & 77 & 3920 & $\mathrm{~F}(?)$ & $1.2^{+}$ \\
6 & 60 & 2050 & $\mathrm{M}(1)$ & $1.1^{+}$ \\
7 & 55 & 1570 & $\mathrm{~F}(1)$ & $2.1^{+}$ \\
\hline
\end{tabular}

(1) confirmé à la recapture ou par le cadavre

- une zone dite "zone entrée" de 70 m de longueur constituée par la partie aval du canal de fuite de Chopolo, à partir du confluent avec la Nive. Cette zone est couverte par une antenne filaire immergée de même longueur.

- une zone dite "zone pont" de $200 \mathrm{~m}$ de longueur environ constituée par le bras de Nive court-circuité par l'aménagement de Chopolo entre les cotes 1100 et.1300 (Fig. 2) et limité en amont par le pont de Jatxou. Cette zone est couverte par une antenne aérienne type boucle.

- un repérage manuel de tous les saumons a été effectué 2 fois par jour (en début de matinée et durant l'après-midi) pendant tout le mois de juin et ensuite une fois par jour jusqu'à la fin de l'expérimentation (16 novembre 1991). On a également procédé à des contrôles fréquents des stations fixes à l'aide d'émetteurs témoins, pour vérifier non seulement le fonctionnement correct de l'appareillage mais également la calibration des zones précédemment définies.

L'écran a été mis en marche immédiatement après le lâcher mais n'a pas fonctionné en permanence pendant toute la durée de l'expérimentation. En effet, on ne pouvait d'une part prendre le risque d'arrêter une grande partie des géniteurs de la Nive dans leur migration d'amontaison et, d'autre part, il était intéressant d'observer le comportement des animaux lorsque l'écran n'était pas électrifié. En conséquence, la séquence de fonctionnement de l'écran se présente, en fonction de l'avancement de la saison de migration, de la façon suivante :

— du 10 juin (J.161) au 9 juillet (J.190) : fonctionnement continu de l'écran ;

— du 9 juillet au 27 septembre (J.270) : fonctionnement de l'écran 1 jour sur 2 ;

— après le 27 septembre : arrêt total de l'écran.

Le piège a fonctionné en permanence pendant toute la durée de l'expérimentation. 


\section{RÉSULTATS}

\subsection{Caractéristiques du milieu}

Le débit fluvial durant la période d'étude (Fig. 4) se caractérise par un faible débit de printemps (10 à $\left.12 \mathrm{~m}^{3} \cdot \mathrm{s}^{-1}\right)$ et d'été $\left(6 \mathrm{~m}^{3} \cdot \mathrm{s}^{-1}\right)$ et par une succession de coups d'eau en automne (20 à $300 \mathrm{~m}^{3} \cdot \mathrm{s}^{-1}$ ). Pendant toute la première partie du suivi et jusqu'au 29 septembre (J.272) environ, la majeure partie du débit de la Nive transite par le canal de Chopolo.

Débit dans lo Nive

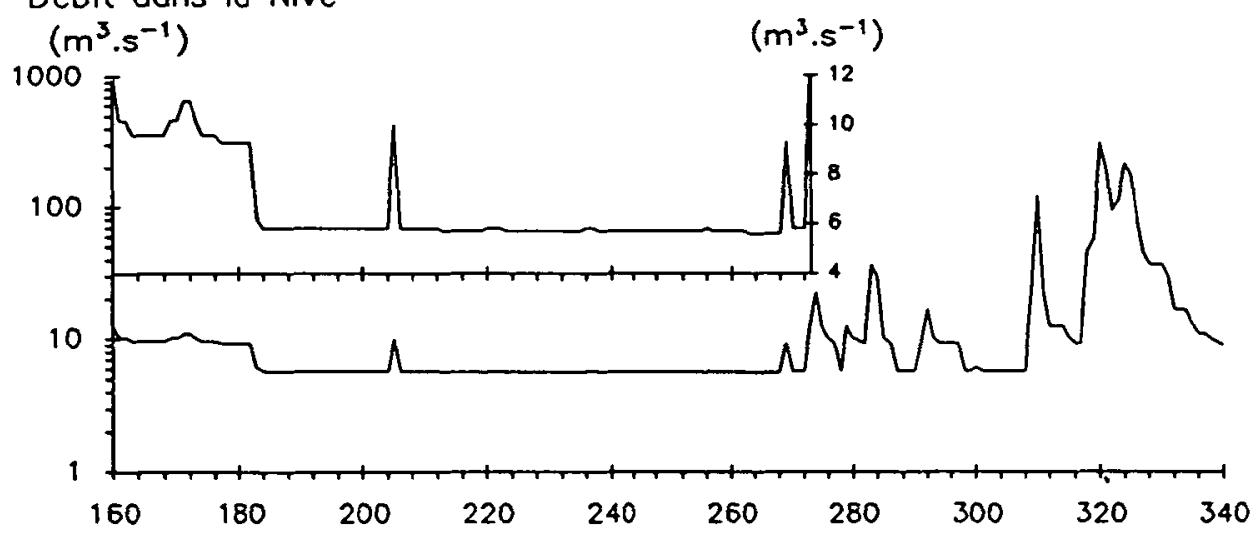

\section{Débit dons le conol de Chopolo \\ (en \% du débit dans la Nive)}

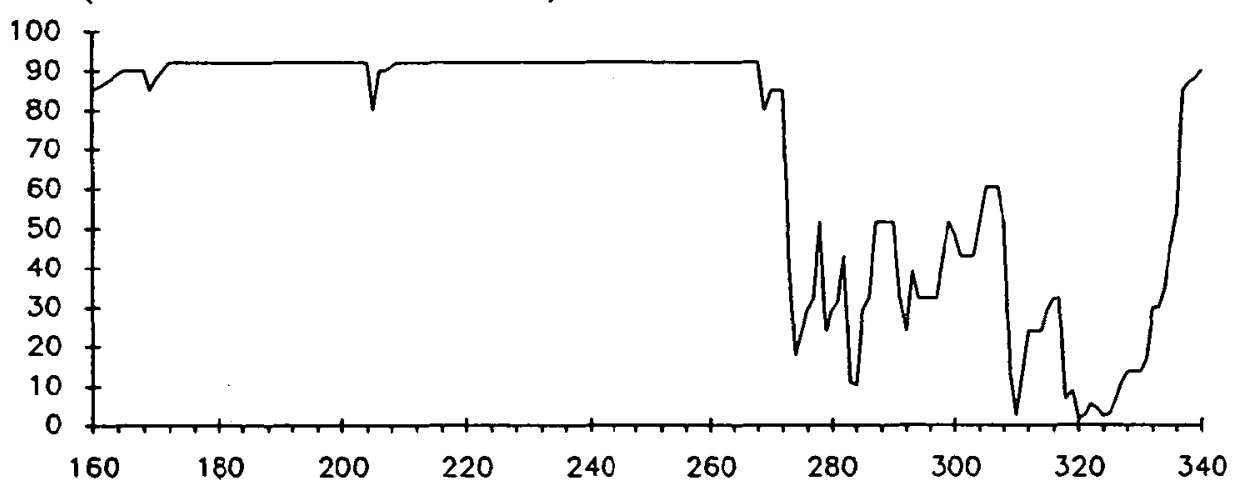

Niveau (cote NGF) ò lo sortie

des turbines (zone écran)

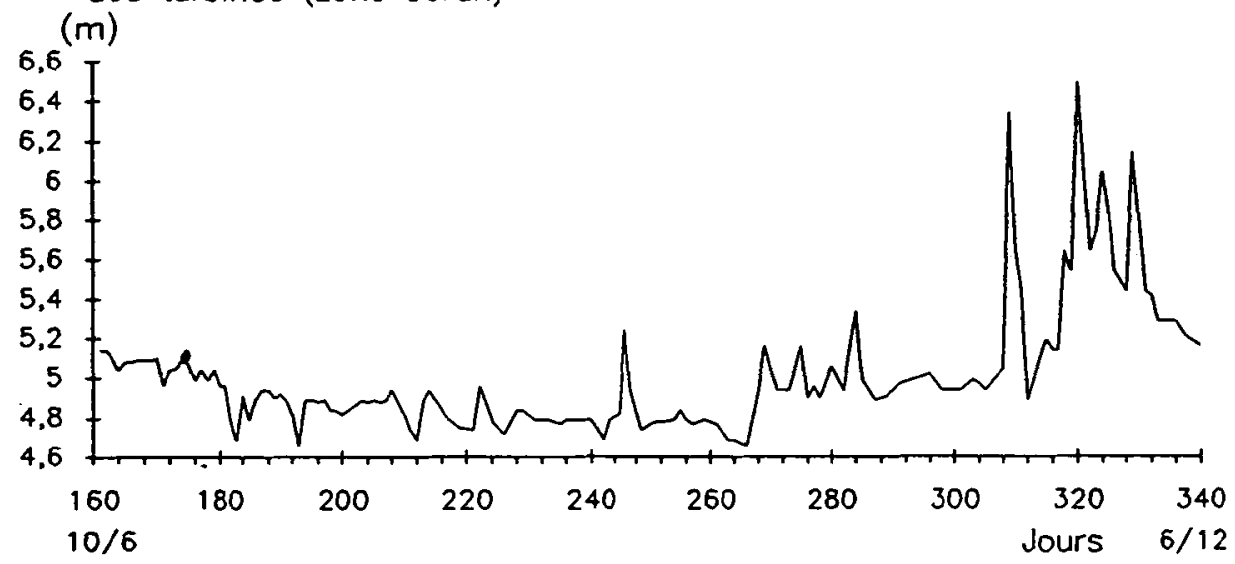

Figure 4 : Caractéristiques hydrologiques pendant la période de surveillance.

Figure 4 : Hydrological characteristics during the observation period. 
En relation avec le faible débit d'étiage on peut également noter (Fig. 5) des températures élevées de l'eau (de $18^{\circ} \mathrm{C}$ à $22^{\circ} \mathrm{C}$ ) pendant une longue période, avec un maximum à $24^{\circ} \mathrm{C}$. Les turbidités très élevées sont la conséquence de pluies d'orage.

La conductivité de l'eau (donnée à température de l'eau) varie entre 160 et $320 \mu{\mathrm{S} . \mathrm{cm}^{-1}}^{-1}$.

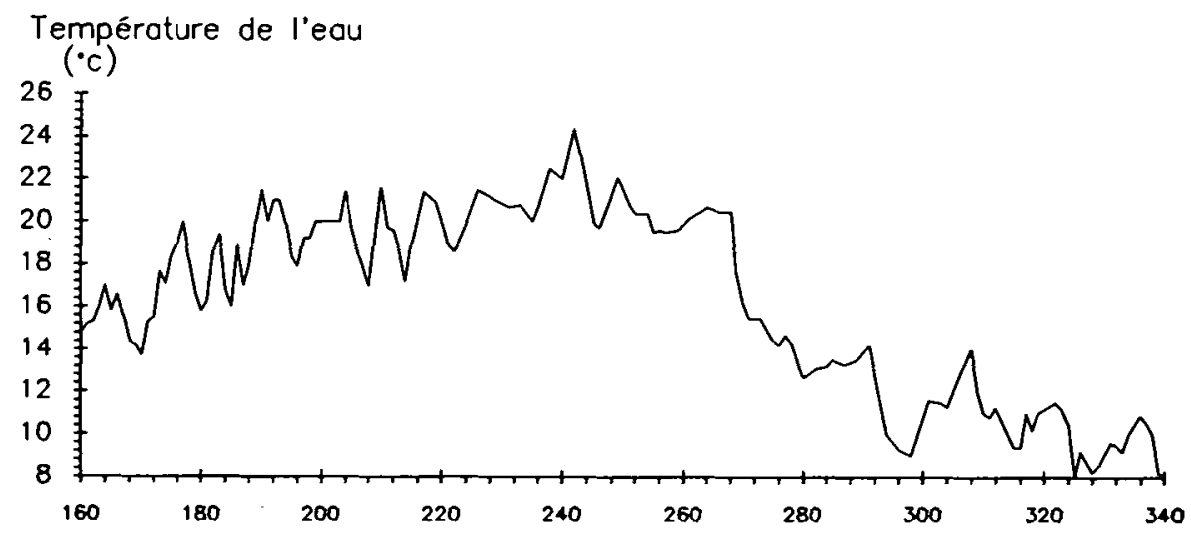

Turbidité de l'eou

(disque de Secchi)
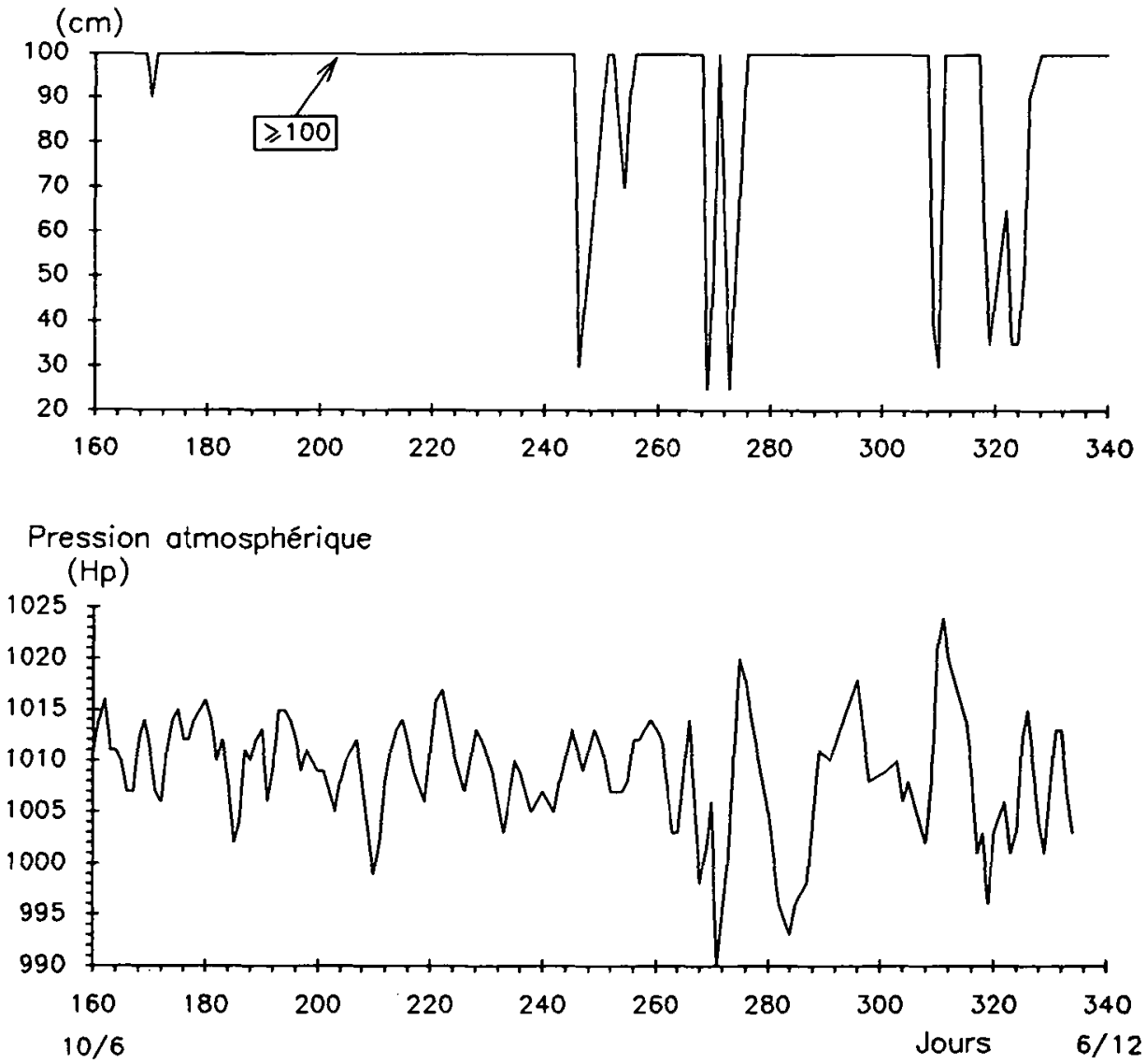

Figure 5 : Caractéristiques physiques de l'eau et pression atmosphérique pendant la période de surveillance.

Figure 5 : Physical characteristics of the water and air-pressure during the observation period. 
Le nombre total des saumons capturés au piège de Chopolo pour l'année 1991 est de 194. La figure 6 précise la fréquence et le nombre des captures (146) pendant la période de surveillance. Les saumons capturés après le 25 septembre (J.268) étaient en grande majorité des castillons de la classe d'âge $1.1^{+}$et présentaient tous une robe très colorée.

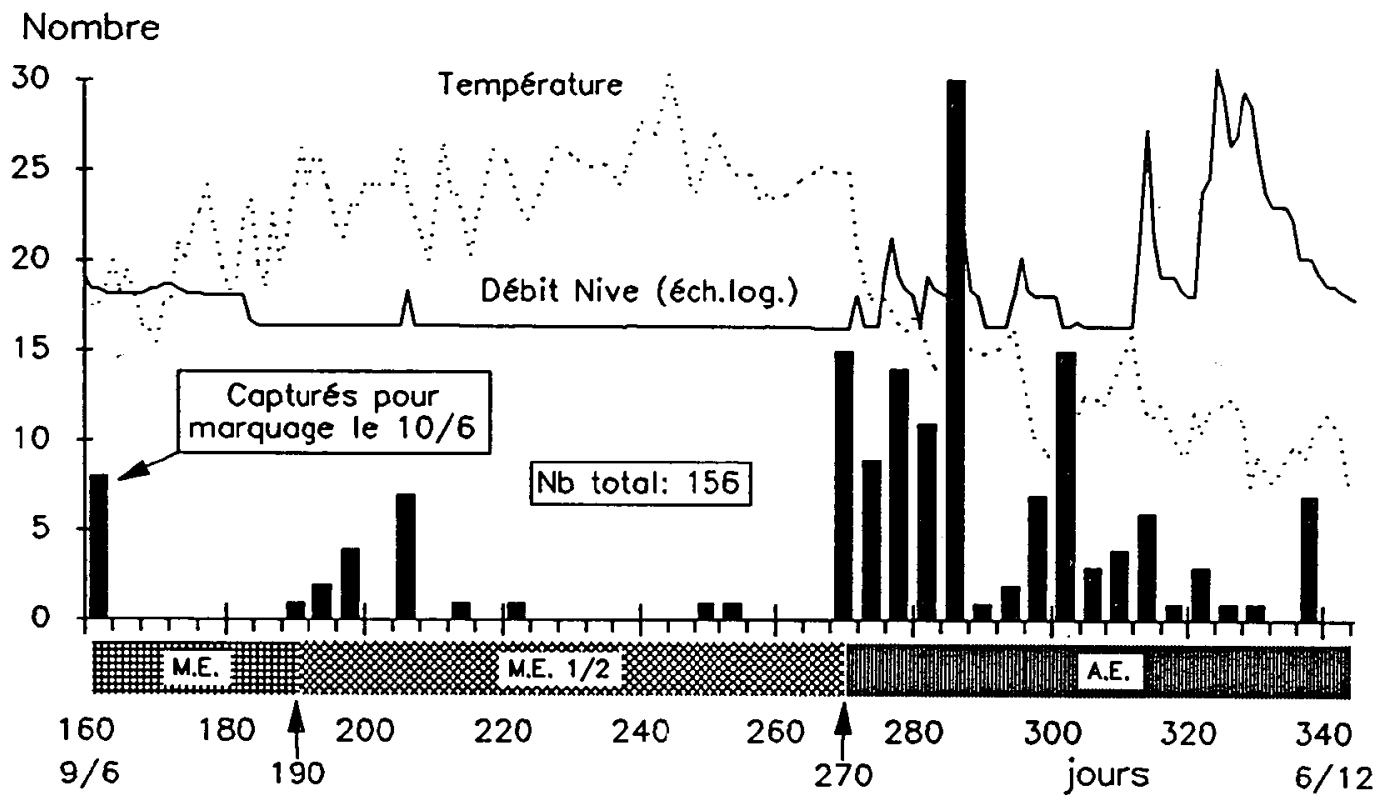

Figure 6 : Captures de saumons au piège durant la période de surveillance (10/06/91 6/12/91).

Figure 6 : Salmon captures in the trap during the observation period (10/06/91 -6/12/91).

\subsection{Etude des déplacements}

Compte tenu de la complexité du réseau constituant la zone expérimentale et des déplacements, on a choisi une méthode graphique pour figurer les trajets effectués par les différents saumons - dans de nombreuses situations on peut parler d'errance ou de vagabondage (Fig. 7 à 22).

Pour préciser la position des saumons à un instant donné, on a pris pour référence la cote zéro au point de lâcher (Fig. 2). Les positions en amont et en aval de ce point ont été respectivement caractérisées par des cotes positives et négatives (exprimées en $\mathrm{m}$ ).

Les déplacements effectués dans le cours principal de la Nive sont figurés par des traits pleins, ceux qui sont effectués dans les canaux d'amenée et de fuite par des pointillés.

Tous les déplacements effectués près du point de lâcher et à l'intérieur de la zone ombrée ont été notés à la cote zéro.

Seuls les enregistrements ou repérages manuels donnant une information supplémentaire ont été précisés : lorsque l'animal a été localisé au même endroit pendant plusieurs jours, on a seulement représenté les repérages correspondant au début et à la fin de cette période. Par ailleurs, il est évident que les déplacements effectués entre 2 repérages-peuvent être sensiblement différents de ceux qui sont figurés par des traits pleins ou pointillés.

Un zoom - par dilatation de l'échelle des temps - a été réalisé sur les déplacements effectués dans le canal de fuite de Chopolo. Sur ces graphes (Fig. 8, 10, 11, 14, 16, 18, 20, 21 et 22) où la plus grande partie des repérages résulte d'enregistrements, on a précisé les temps de présence dans la zone écran. Lorsque 2 périodes consécutives de présence sont enregistrées à la même cote (zone écran ou zone entrée), le parcours de l'animal entre 2 périodes consécutives n'est représenté par aucun trait : ceci signifie qu'aucun repérage ne permet de préciser le trajet effectué entre 2 périodes : 
Le saumon $n^{\circ} 1$ a fait preuve d'une grande activité au début de la période de surveillance (Fig. 7 et 8 ). Deux jours après le marquage, il s'est engagé dans le canal de fuite de Chopolo. Apparemment repoussé par l'écran, il a fait durant quatre jours plusieurs allers et retours entre la Nive (zone pont ou confluent du canal de fuite et du bras court-circuité) et la zone écran. Les enregistrements continus relevés pour la zone pont montrent qu'il n'est pas remonté en amont du pont de Jaxou. II est mort 9 jours après le marquage. La récupération du cadavre, toujours porteur de l'émetteur, n'a pas permis d'en définir les causes.

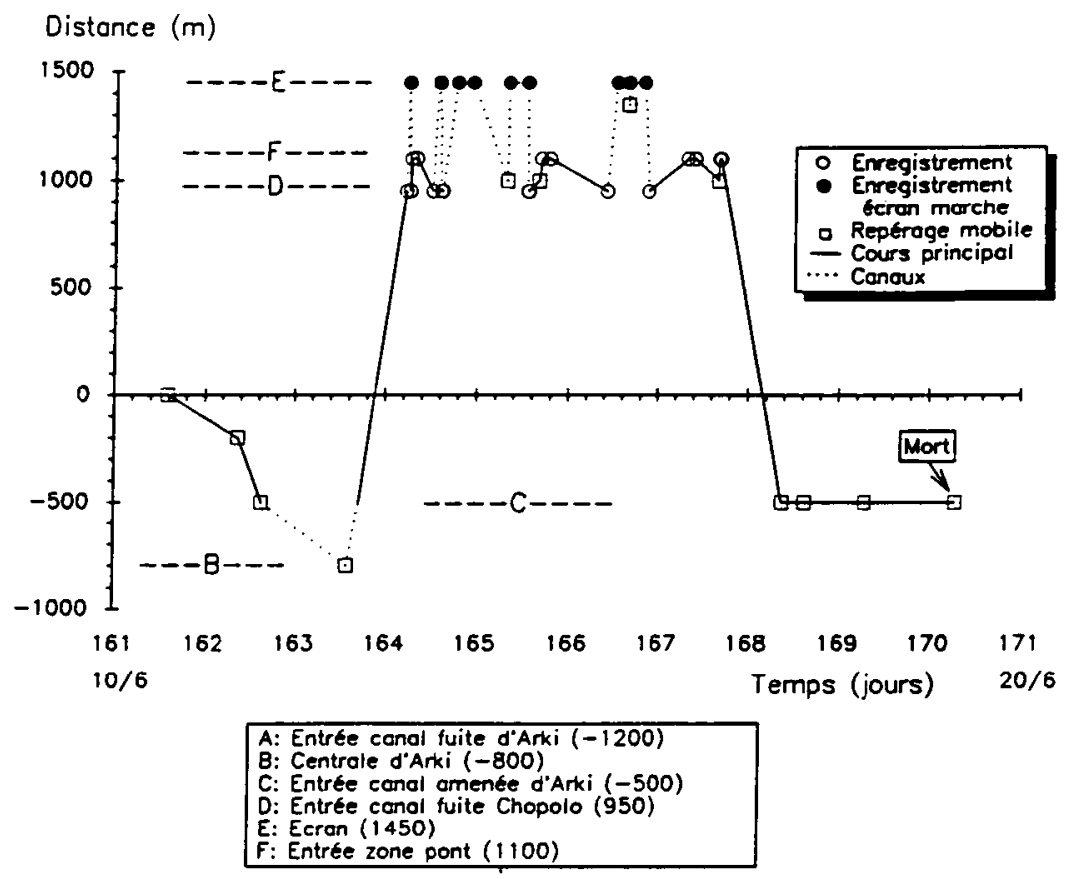

Figure 7 : Déplacements du saumon $n^{\circ} 1$ dans la zone expérimentale.

Figure 7 : Movements of salmon $n^{\circ} 1$ in the experimental zone.

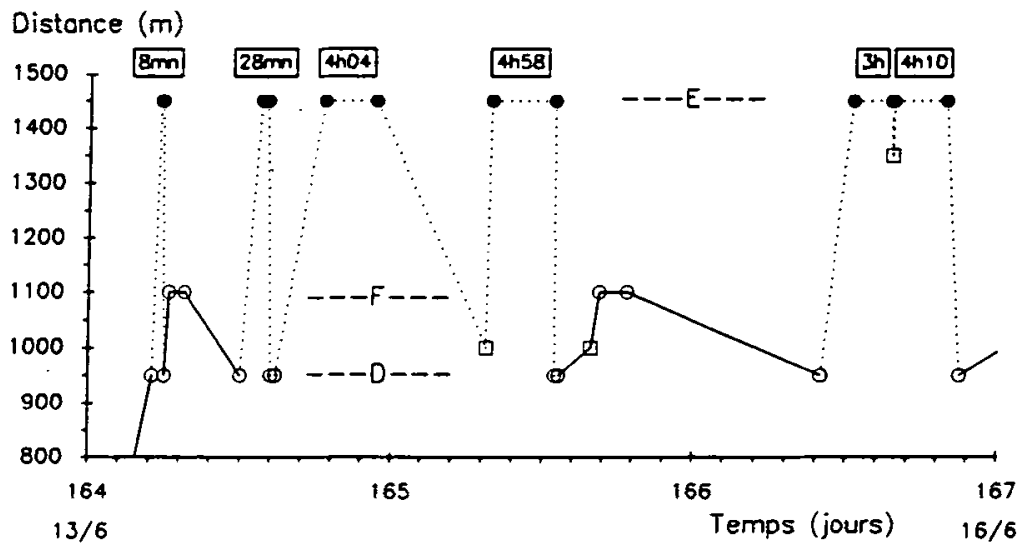

Figure 8 : Déplacements du saumon $n^{\circ} 1$ dans le canal de fuite de Chopolo.

Figure 8 : Movements of salmon $n^{\circ} 1$ in the Chopolo channel discharge. 
Le saumon $n^{\circ} 2$ a vagabondé dans la Nive et le canal d'amenée d'Arki pendant 45 jours environ, avant de pénétrer, fin juillet, dans le canal de fuite de Chopolo (Fig. 9). Bien que l'écran n'ait pas été en fonctionnement, il n'a pas franchi la passe et est retourné dans la Nive jusqu'à l'automne. Fin septembre et début novembre, il a effectué de nouveau deux séries de tentatives de remontée dans le canal de fuite de Chopolo sans emprunter la passe, alors que l'écran était toujours à l'arrêt (Fig. 9, 10 et 11). Ces tentatives ont été entrecoupées de longues périodes d'errance avec une dévalaison importante jusqu'au barrage de la Lyonnaise des Eaux. Il est passé à l'amont de Chopolo par le lit principal le 16 novembre (J.320) à la faveur d'un coup d'eau.

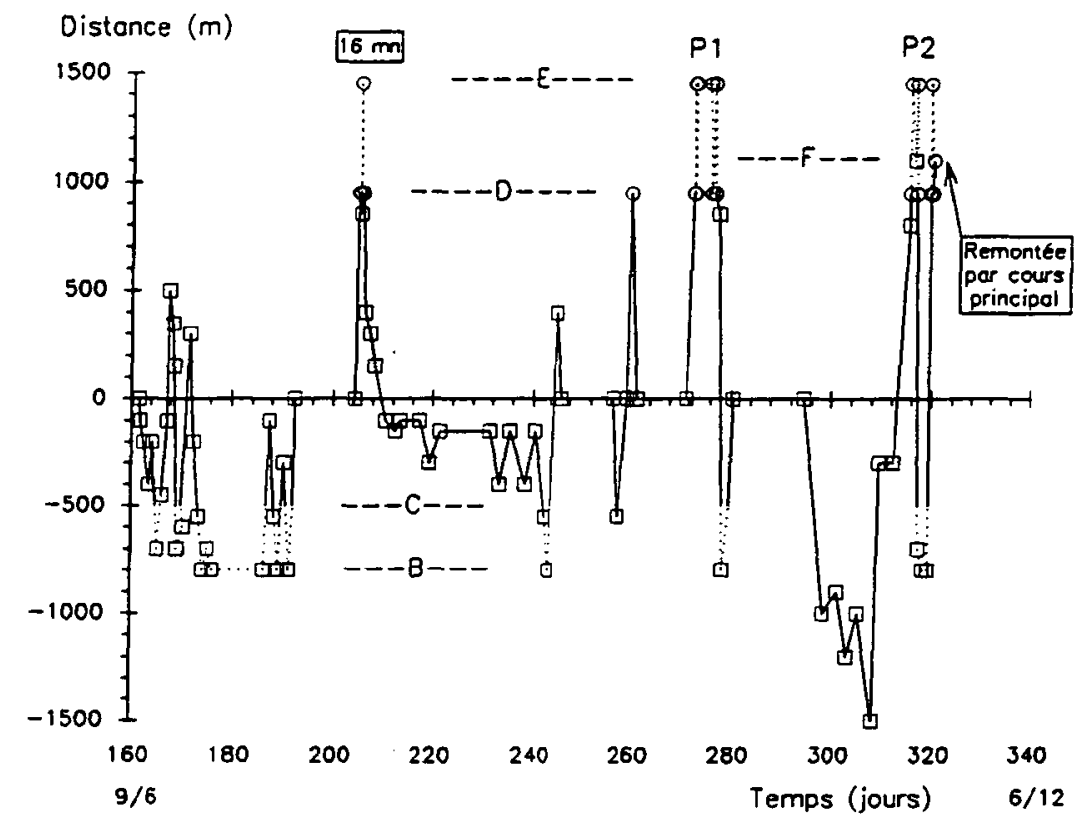

Figure 9 : Déplacements du saumon $n^{\circ} 2$ dans la zone expérimentale.

Figure 9 : Movements of salmon $n^{\circ} 2$ in the experimental zone.

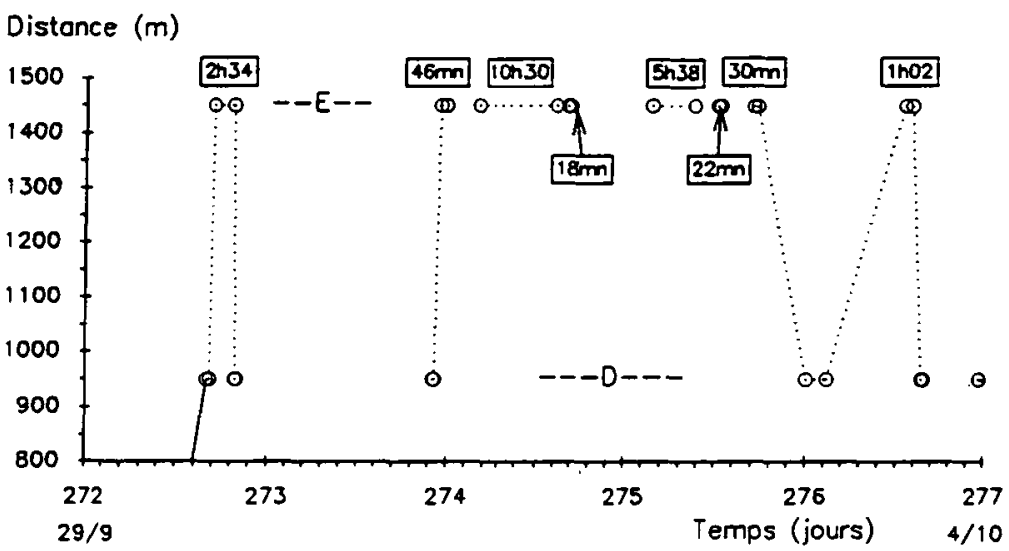

Figure 10 : Déplacements du saumon $n^{\circ} 2$ dans le canal de fuite de Chopolo pendant la période $\mathbf{P 1}$.

Figure 10 : Movements of salmon $n^{\circ} 2$ in the Chopolo channel discharge during the period P1. 


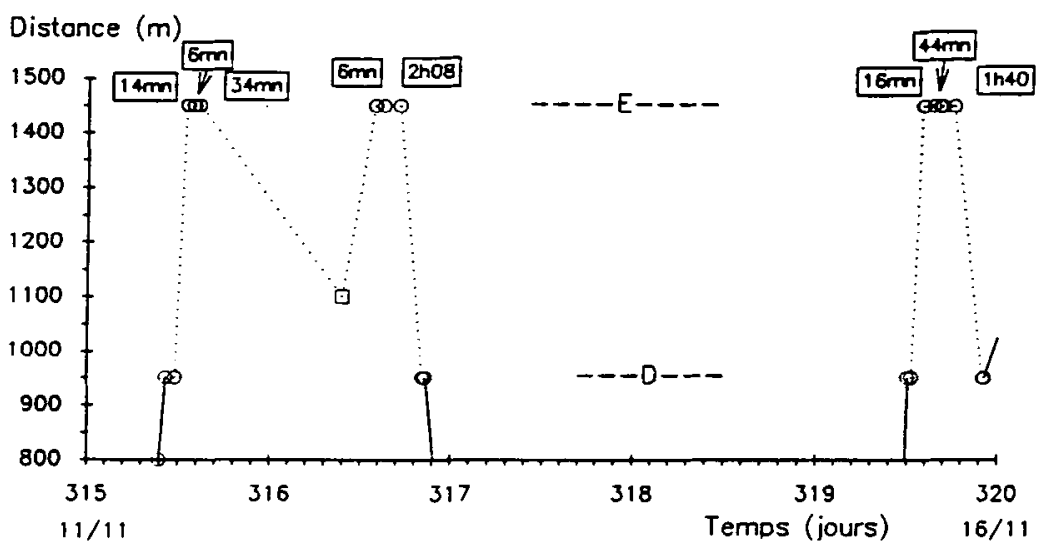

Figure 11 : Déplacements du saumon $n^{\circ} 2$ dans le canal de fuite de Chopolo pendant la période $\mathbf{P 2}$.

Figure 11 : Movements of salmon $n^{\circ} 2$ in the Chopolo channel discharge during the period P2.

Le saumon $\mathbf{n}^{\circ} \mathbf{3}$ a vagabondé dans la Nive et le canal d'amenée d'Arki pendant 92 jours avant de mourir début septembre (Fig. 12). Il est remonté à plusieurs reprises à l'entrée du canal de fuite de Chopolo et dans le bras court-circuité, mais n'a jamais emprunté le canal de fuite jusqu'à l'écran électrique. Son cadavre n'a pu être récupéré et les causes de sa mort restent inconnues.

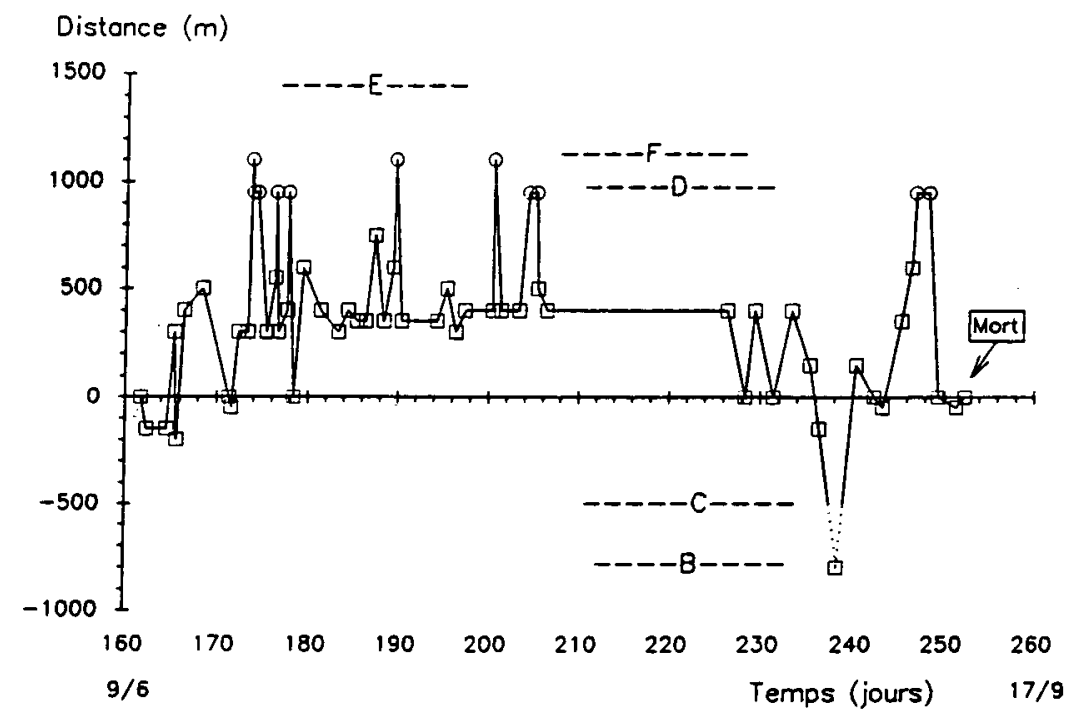

Figure 12 : Déplacements du saumon $n^{\circ} 3$ dans la zone expérimentale.

Figure 12 : Movements of salmon $n^{\circ} 3$ in the experimental zone.

Le saumon $n^{\circ} 4$ a vagabondé pendant 140 jours environ dans la partie basse de la zone d'expérimentation (gravière et canal de fuite de la centrale d'Arki) (Fig. 13), avant de remonter très rapidement le 8 novembre (J.312) à la faveur d'un coup d'eau. II a alors fait, durant $24 \mathrm{~h}$, une série de tentatives de remontée par le canal de fuite de Chopolo (Fig. 14), a pénétré plusieurs fois dans la zone écran (écran à l'arrêt), mais n'a pas emprunté la passe et est finalement remonté par le lit principal. 


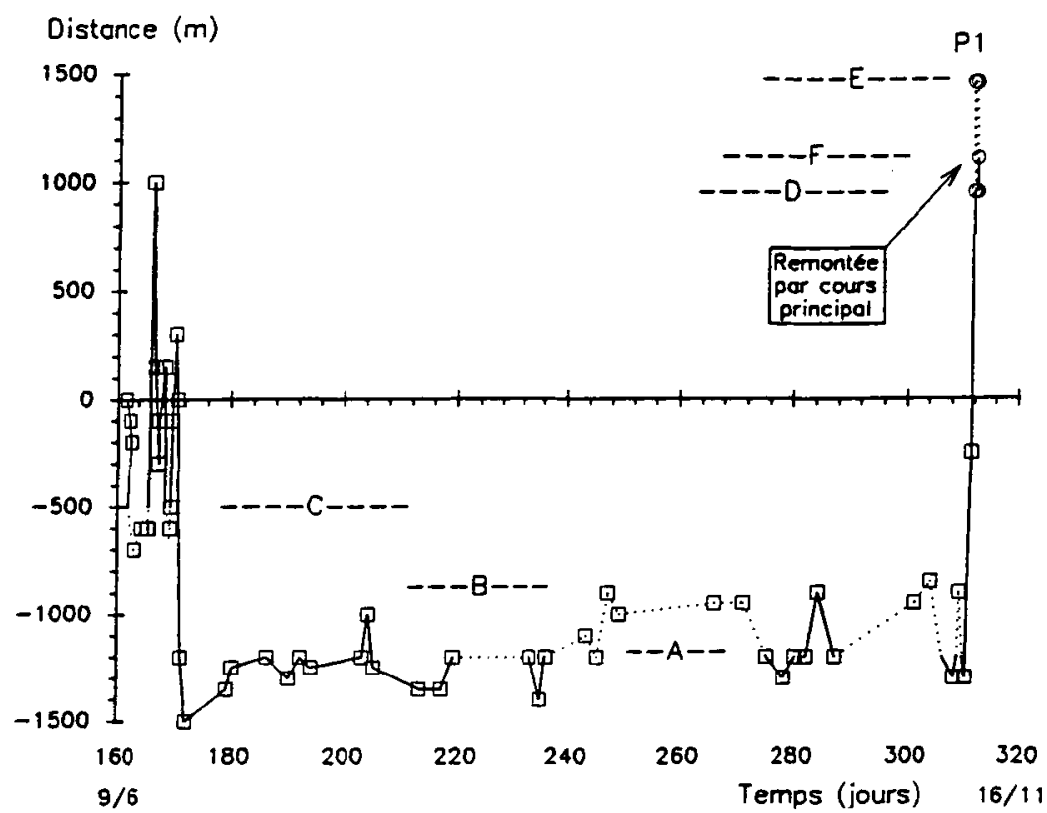

Figure 13 : Déplacements du saumon $n^{\circ} 4$ dans la zone expérimentale.

Figure 13 : Movements of salmon $n^{\circ} 4$ in the experimental zone.

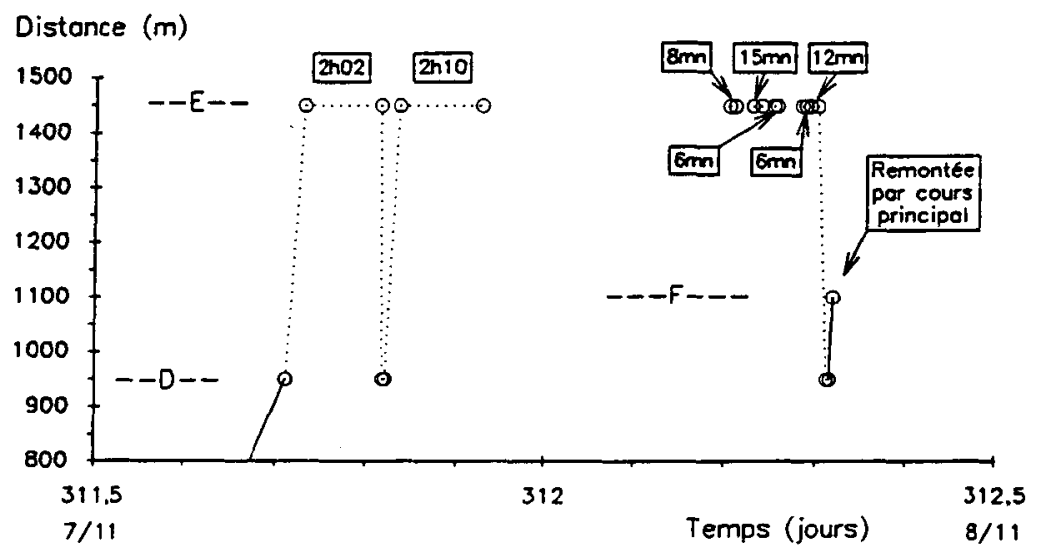

Figure 14 : Déplacements du saumon $n^{\circ} 4$ dans le canal de fuite de Chopolo pendant la période $\mathrm{P1}$.

Figure 14 : Movements of salmon $n^{\circ} 4$ in the Chopolo channel discharge during the period P1.

Le saumon $\mathbf{n}^{\circ} \mathbf{5}$ a fait une tentative de remontée par le canal de fuite de Chopolo début juillet, soit 26 jours après le marquage (Fig. 15). II s'est présenté à plusieurs reprises devant l'écran en marche au cours de la même journée (Fig. 16), puis a fait une incursion dans le bras court-circuité avant de redévaler. Vient ensuite une longue période d'attente de 92 jours dans le canal d'amenée d'Arki puis une remontée rapide le 10 octobre (J.283) par le lit principal lors d'un coup d'eau (37 $\left.\mathrm{m}^{3} . \mathrm{s}^{-1}\right)$. 


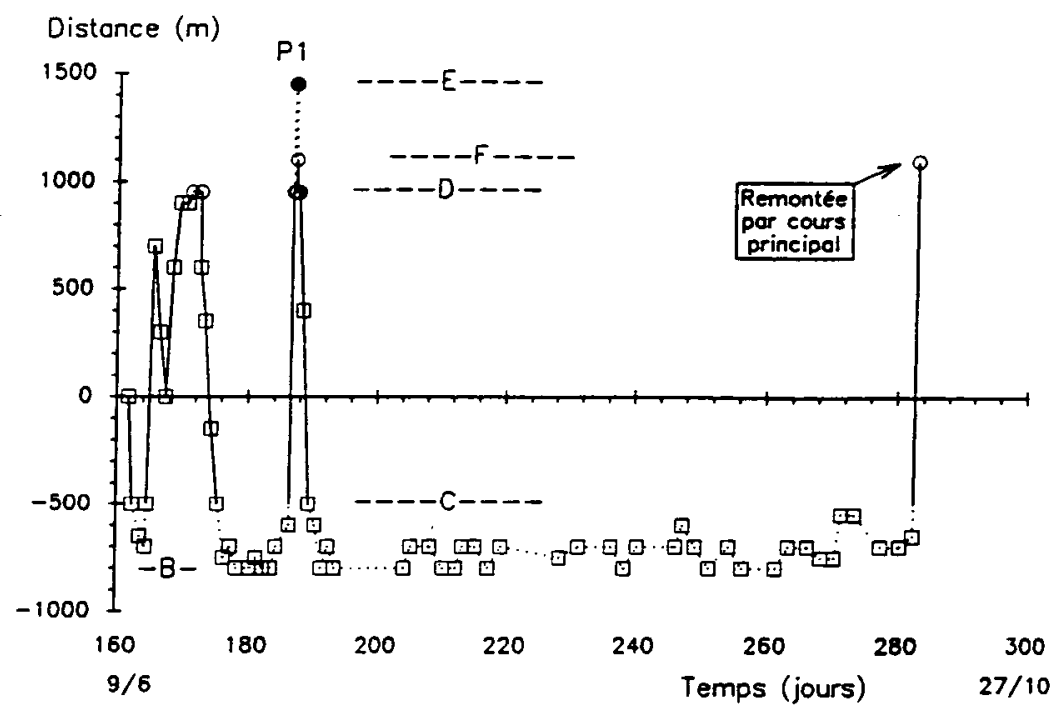

Figure 15 : Déplacements du saumon $n^{\circ} 5$ dans la zone expérimentale.

Figure 15 : Movements of salmon $n^{\circ} 5$ in the experimental zone.

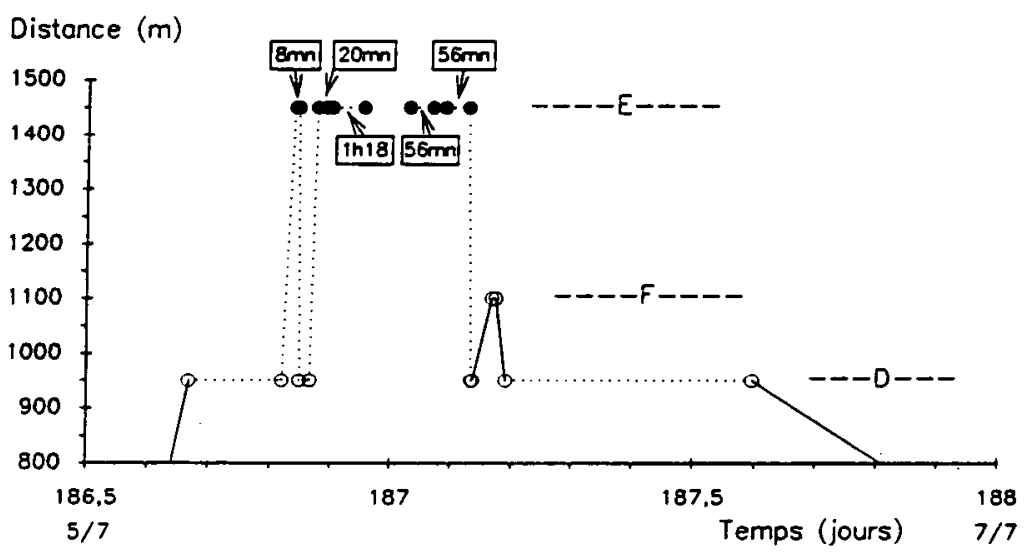

Figure 16 : Déplacements du saumon $n^{\circ} 5$ dans le canal de fuite de Chopolo pendant la période P1.

Figure 16 : Movements of salmon $n^{\circ} 5$ in the Chopolo channel discharge during the period P1.

Le saumon $n^{\circ} 6$ a vagabondé dans la Nive jusqu'à début octobre (114 jours après marquage) (Fig. 17). II a fait durant cette période de nombreux allers et retours entre un site d'attente privilégié et l'entrée du canal de fuite de Chopolo en pénétrant à deux reprises dans le bras court-circuité (zone pont) sans toutefois dépasser le pont de Jaxou. Il a finalement emprunté la passe de Chopolo le 5 octobre (J.278), alors que l'écran électrique était à l'arrêt et a été recapturé. Ce passage a été précédé d'un stationnement de 4 jours dans la zone écran (écran en permanence à l'arrêt) (Fig. 18). 


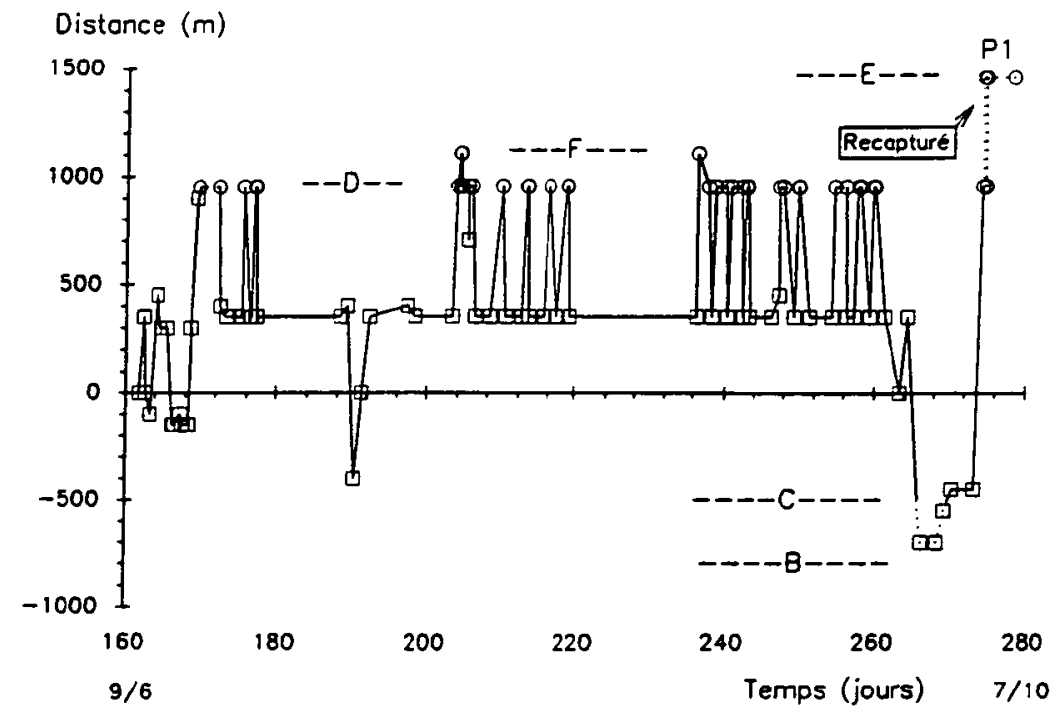

Figure 17 : Déplacements du saumon $n^{\circ} 6$ dans la zone expérimentale.

Figure 17 : Movements of salmon $n^{\circ} 6$ in the experimental zone.

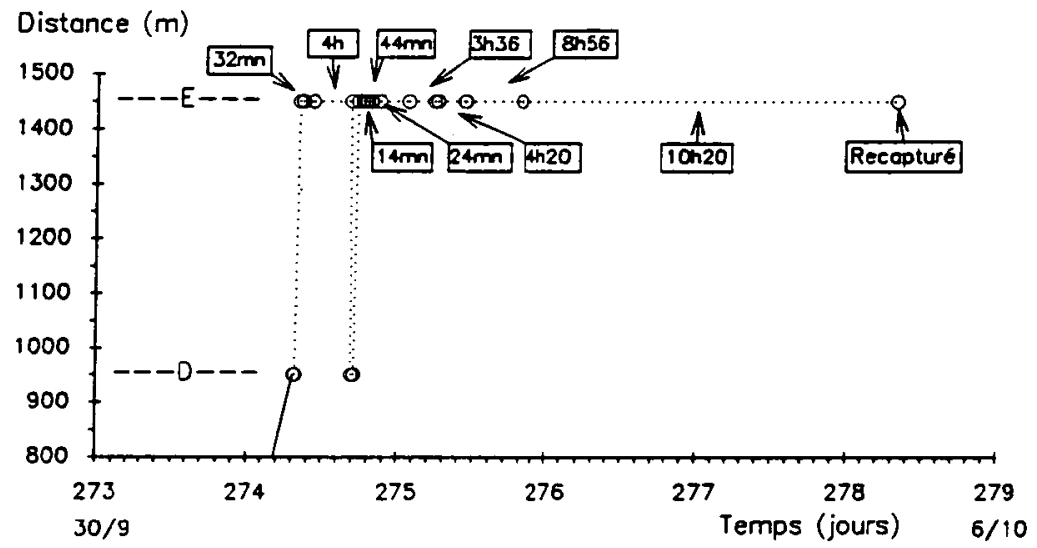

Figure 18 : Déplacements du saumon $n^{\circ} 6$ dans le canal de fuite de Chopolo pendant la période $\mathbf{P 1}$.

Figure 18 : Movements of salmon $n^{\circ} 6$ in the Chopolo channel discharge during the period P1.

Le saumon $n^{\circ} 7$ est celui qui a manifesté le plus fréquemment son besoin d'amontaison (Fig. 19). Il est monté très rapidement jusqu'à la zone écran (écran en marche) (Fig. 20), a vagabondé dans la Nive pendant une dizaine de jours puis a stationné durant 42 jours dans le canal de fuite de Chopolo (Fig. 21), pénétrant à plusieurs reprises dans la zone écran (écran parfois en marche, parfois à l'arrêt). Après une deuxième période d'une vingtaine de jours de déplacement dans la Nive, il a emprunté la passe de Chopolo (écran à l'arrêt) et a été recapturé le 28 septembre (J.271) après deux jours d'allers et retours entre la zone écran et l'entrée du canal de fuite (Fig. 22). 


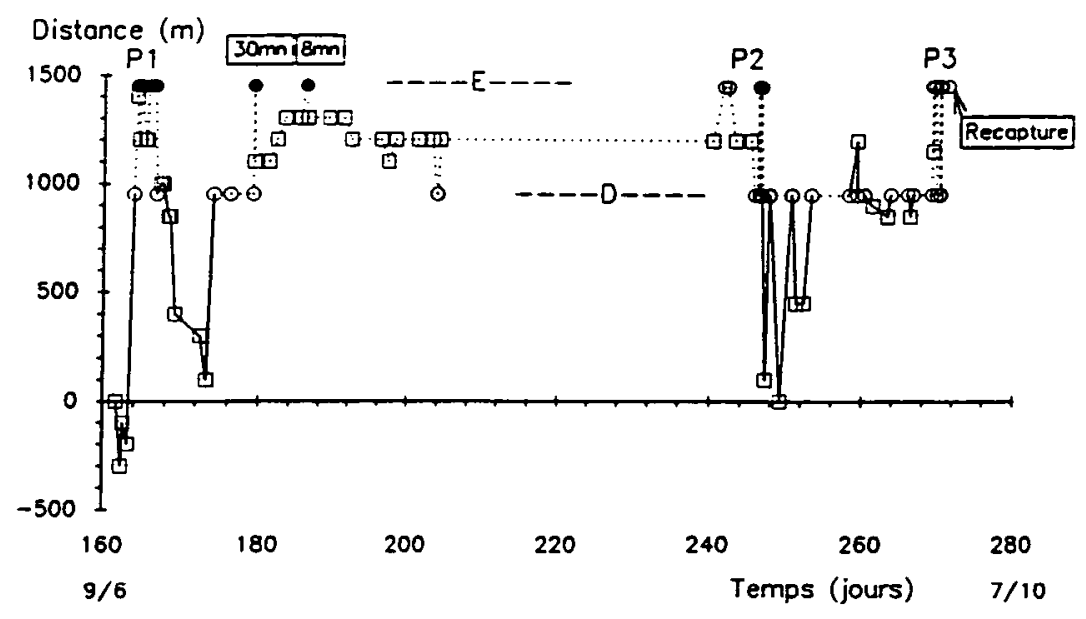

Figure 19 : Déplacements du saumon $n^{\circ} 7$ dans la zone expérimentale.

Figure 19 : Movements of salmon $n^{\circ} 7$ in the experimental zone.

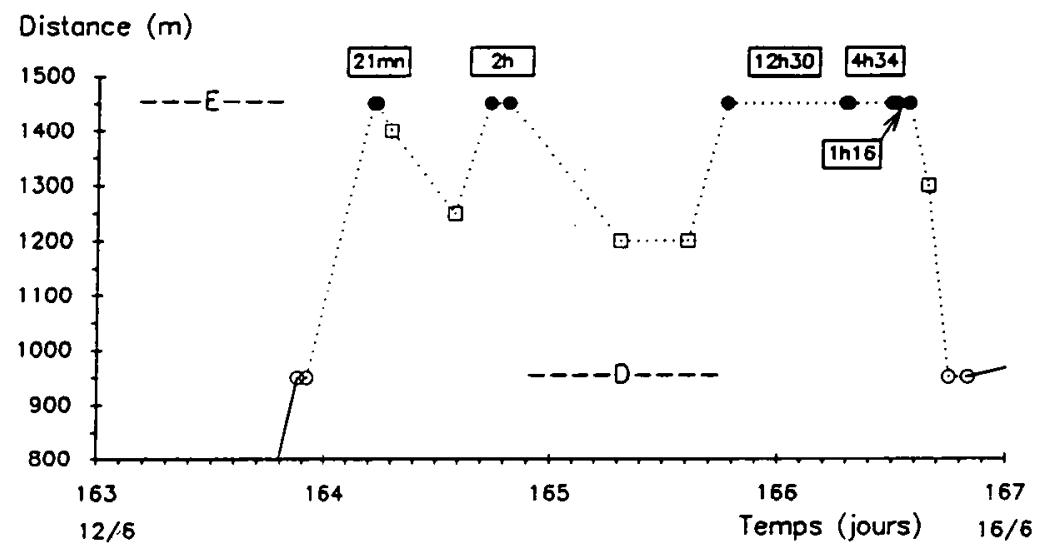

Figure 20 : Déplacements du saumon $n^{\circ} 7$ dans le canal de fuite de Chopolo pendant la période $\mathbf{P 1}$.

Figure 20 : Movements of salmon $n^{\circ} 7$ in the Chopolo channel discharge during the period P1.

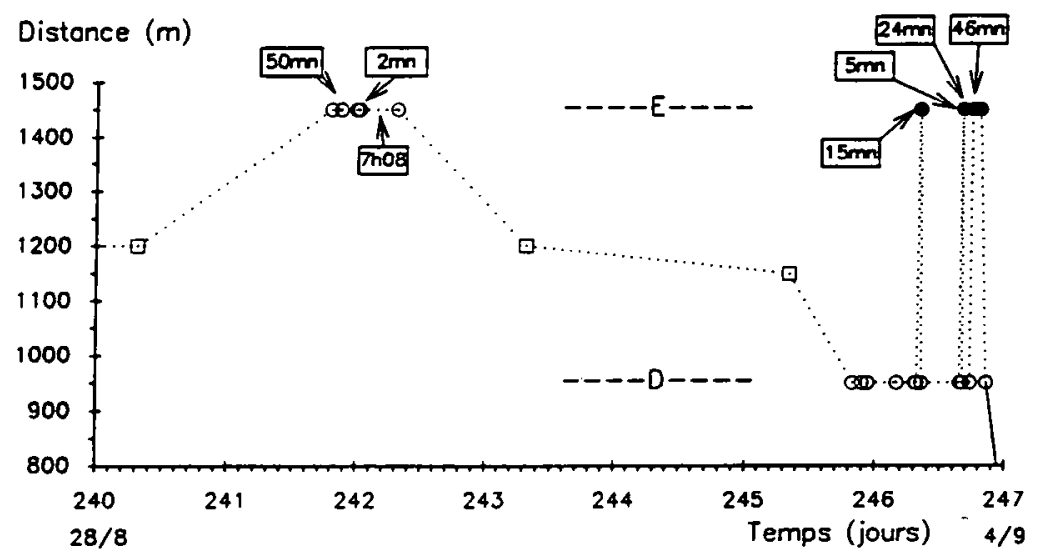

Figure 21 : Déplacements du saumon $n^{\circ} 7$ dans le canal de fuite de Chopolo pendant la période $\mathbf{P 2}$.

Figure 21 : Movements of salmon $n^{\circ} 7$ in the Chopolo channel discharge during the period P2. 


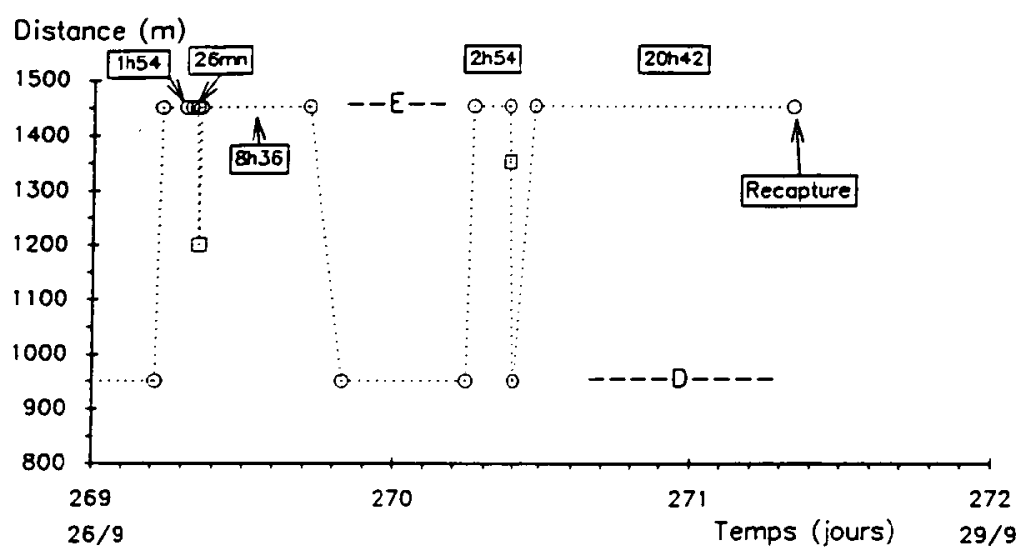

\section{Figure 22 : Déplacements du saumon $n^{\circ} 7$ dans le canal de fuite de Chopolo pendant la période $\mathbf{P 3}$.}

Figure 22 : Movements of salmon $n^{\circ} 7$ in the Chopolo channel discharge during the period P3.

De l'analyse des résultats présentés ci-dessus, on peut tirer divers enseignements : des enseignements d'ordre général concernant le comportement migratoire dans une zone à obstacles et des enseignements plus particulièrement liés à l'utilisation d'un écran électrique.

\subsubsection{Comportement migratoire dans la Nive aval}

- L'hétérogénéité des comportements est évidente malgré des conditions de capture et de marquage identiques : il n'y a pas 2 saumons qui ont séjourné dans les mêmes endroits ou effectué les mêmes parcours au même moment, sice n'est immédiatement après le marquage (légère dévalaison) ou juste avant la remontée finale.

- L'activité est principalement liée aux variations de débit (mouvements accrus sur les coups d'eau) et à la température de l'eau : on n'observe pas de mouvements de grande amplitude des saumons marqués et le nombre de captures des saumons non marqués est très faible de fin juillet à fin septembre - J.208 à J.268 environ (Fig. 6) - lorsque la température moyenne dépasse $20^{\circ} \mathrm{C}$. Si l'on peut observer que les déplacements les plus importants se produisent souvent lorsque la turbidité est forte (surtout en automne), il est impossible d'affirmer que ce paramètre ait une influence directe sur l'activité, puisque il est à l'évidence lié aux variations de débit.

Par la comparaison rapide des courbes, on ne peut non plus affirmer que l'intensité des déplacements est liée à la pression atmosphérique ; une étude plus fine sortant du cadre de cette étude serait nécessaire. Cependant, il a été fréquemment observé, surtout en été et au début de l'automne, que l'activité des saumons marqués augmentait pendant les périodes orageuses sans pluie ou accompagnées de faibles précipitations et donc n'entraînant pas de changement sensible du débit.

- Les saumons ne restent pas bloqués dans un canal de fuite d'une façon préférentielle. Ils peuvent également séjourner très longtemps (plusieurs mois) dans le cours principal de la rivière, dans des sites ou abris privilégiés.

- Il est évident que la capture a stoppé momentanément la migration déclenchée par le coup d'eau du 10 juin mais on ne peut affirmer que, par la suite, le stress consécutif au marquage et (ou) à la capture (effet de mémorisation ?) soit seul responsable de l'arrêt de migration pendant l'été au niveau d'Ustaritz. En effet, alors que les captures au piège de Chopolo ont été très faibles en été, de nombreux saumons ont été capturés à partir du 25 septembre (J.268) (Fig. 6). Tous étaient très colorés et avaient donc séjourné en eau douce depuis plusieurs semaines, voire plusieurs mois, bien qu'ils aient eu la possibilité de remonter par la passe de Chopolo bien avant cette date puisque l'écran était à l'arrêt 1 jour sur 2 depuis 
le 10 juillet. L'efficacité de cette passe fonctionnant dans ces conditions ayant été demontrée (GOSSET, 1989), on peut supposer que ces poissons n'ont probablement pas cherché à l'emprunter pendant cette période estivale (10 juillet - 25 septembrè). L'avancement de la saison de migration et les conditions climatiques (température basse.et coups d'eau de fin septembre, d'octobre et de novembre) sont très probablement les principaux facteurs qui ont déclenché la reprise de migration (phase finale) vers les frayères (Tabl. II).

\section{Tableau II : Conditions de débit et de température lors de la remontée finale, vers l'amont de la zone expérimentale.}

Table II : Temperature and flow conditions during the final ascend migration, upstream of the experimental zone.

\begin{tabular}{|c|c|c|c|c|c|c|}
\hline $\begin{array}{c}N^{\circ} \\
\text { saumon }\end{array}$ & Date de passage & Lieu de passage & $\begin{array}{l}\text { Débit } \\
\text { canal fuite } \\
\left(m^{3} \cdot s^{-1}\right)\end{array}$ & $\begin{array}{l}\text { Débit bras } \\
\text { C.c. Nive } \\
\left(\mathrm{m}^{3} \cdot \mathrm{s}^{-1}\right)\end{array}$ & $\begin{array}{c}\text { Déb.canal/ } \\
\text { déb. bras c.c. } \\
(\%)\end{array}$ & $\begin{array}{c}\text { Température } \\
\left({ }^{\circ} \mathrm{C}\right)\end{array}$ \\
\hline 1 & Mort 19/06 (J.170) & & & & & \\
\hline 2 & 16/11 (J.320) & Bras c.c. Nive & 5 & 304 & 1,6 & 11 \\
\hline 3 & Mort $9 / 09$ (J.252) & & & & & \\
\hline 4 & $8 / 11$ (J.312) & Bras c.c. Nive & 3 & 13 & 23 & 11,3 \\
\hline 5 & $10 / 10$ (J.283) & Bras c.c. Nive & 4 & 37 & 11 & 13,1 \\
\hline 6 & $5 / 10(J .278)$ & Canal (passe) & 3 & 6 & 50 & 14,3 \\
\hline 7 & 28/09 (J.271) & Canal (passe) & 5 & 6 & 83 & 15,5 \\
\hline
\end{tabular}

On peut, en conséquence, penser que le retard occasionné par les obstacles, dans la zone d'Ustaritz cette année-là a été très variable suivant la date d'arrivée des saumons dans ce secteur : relativement faible pour les castillons qui sont remontés de l'estuaire en fin d'été et en automne, beaucoup plus important pour ceux qui sont arrivés au printemps et n'ont pas réussi à passer avant l'apparition des conditions climatiques défavorables de l'été.

\subsubsection{Effet de l'écran électrique}

Plusieurs conclusions peuvent être tirées de l'analyse du comportement des trois poissons qui se sont présentés devant l'écran électrique en fonctionnement :

- l'efficacité répulsive de l'écran, déjà mise en évidence les années précédentes sur le même site (GOSSET, 1989), se confirme puisque aucun des poissons n'a franchi l'écran au cours des diverses approches (Tabl. III) que chacun d'eux a effectuées pendant l'étude tous les saumons non marqués contrôlés pendant cette même période ont également été piégés les jours où l'écran était à l'arrêt - ; 
Tableau III : Mouvements des saumons dans la zone écran.

M.E. : marche écran ; M.E. $1 / 2$ : marche écran 1 jour sur 2

A.E. : arrêt écran

Table III : Movements of the salmon in the screen zone.

M.E. : screen on ; M.E. $1 / 2$ : screen on every two days

A.E. : screen off

\begin{tabular}{|c|c|c|c|c|c|}
\hline \multirow{2}{*}{$\begin{array}{c}\mathrm{N}^{\circ} \\
\text { des } \\
\text { saumons }\end{array}$} & \multicolumn{2}{|c|}{$\begin{array}{l}\text { Présence dans la zone écran } \\
\text { (écran en fonctionnement) }\end{array}$} & \multicolumn{2}{|c|}{$\begin{array}{l}\text { Présence dans la zone écran } \\
\text { (écran à l'arrêt) }\end{array}$} & \multirow{2}{*}{$\begin{array}{l}\text { Type de } \\
\text { fonct. }\end{array}$} \\
\hline & $\begin{array}{l}\text { Nombre de } \\
\text { périodes }\end{array}$ & $\begin{array}{l}\text { Durée des périodes } \\
\text { min. - max. }\end{array}$ & $\begin{array}{l}\text { Nombre de } \\
\text { périodes }\end{array}$ & $\begin{array}{l}\text { Durée des périodes } \\
\text { min. - max. }\end{array}$ & \\
\hline 1 & 6 & $8 m n-4 h 58$ & 0 & & M.E. \\
\hline 2 & 0 & & 17 & $6 m n-10 h 30$ & $\begin{array}{l}\text { M.E. } 1 / 2 \\
\text { A.E. }\end{array}$ \\
\hline 3 & 0 & & 0 & & \\
\hline 4 & 0 & & 7 & $6 \mathrm{mn}-2 \mathrm{~h} 10$ & A.E. \\
\hline 5 & 5 & $8 \mathrm{mn}-1 \mathrm{~h} 18$ & 0 & & M.E. \\
\hline 6 & 0 & & 9 & $14 m n-10 h 20$ & A.E. \\
\hline 7 & 11 & $2 m n-12$ h 30 & 8 & $2 m n-20 h 42$ & $\begin{array}{l}\text { M.E. } \\
\text { M.E. } 1 / 2 \\
\text { A.E. }\end{array}$ \\
\hline
\end{tabular}

- le passage dans le champ électrique de l'écran ne semble pas choquer les poissons ni perturber de façon anormale leur comportement. En effet, aucun n'a manifesté de réaction de dévalaison définitive après répulsion et ils ont tous effectué plusieurs prospections dans le champ électrique. Ils ont par ailleurs tous effectué après passage dans la zone d'influence de l'écran, des déplacements dans la Nive semblables à ceux des poissons n'ayant pas pénétré dans le champ électrique (Fig. 23) ;

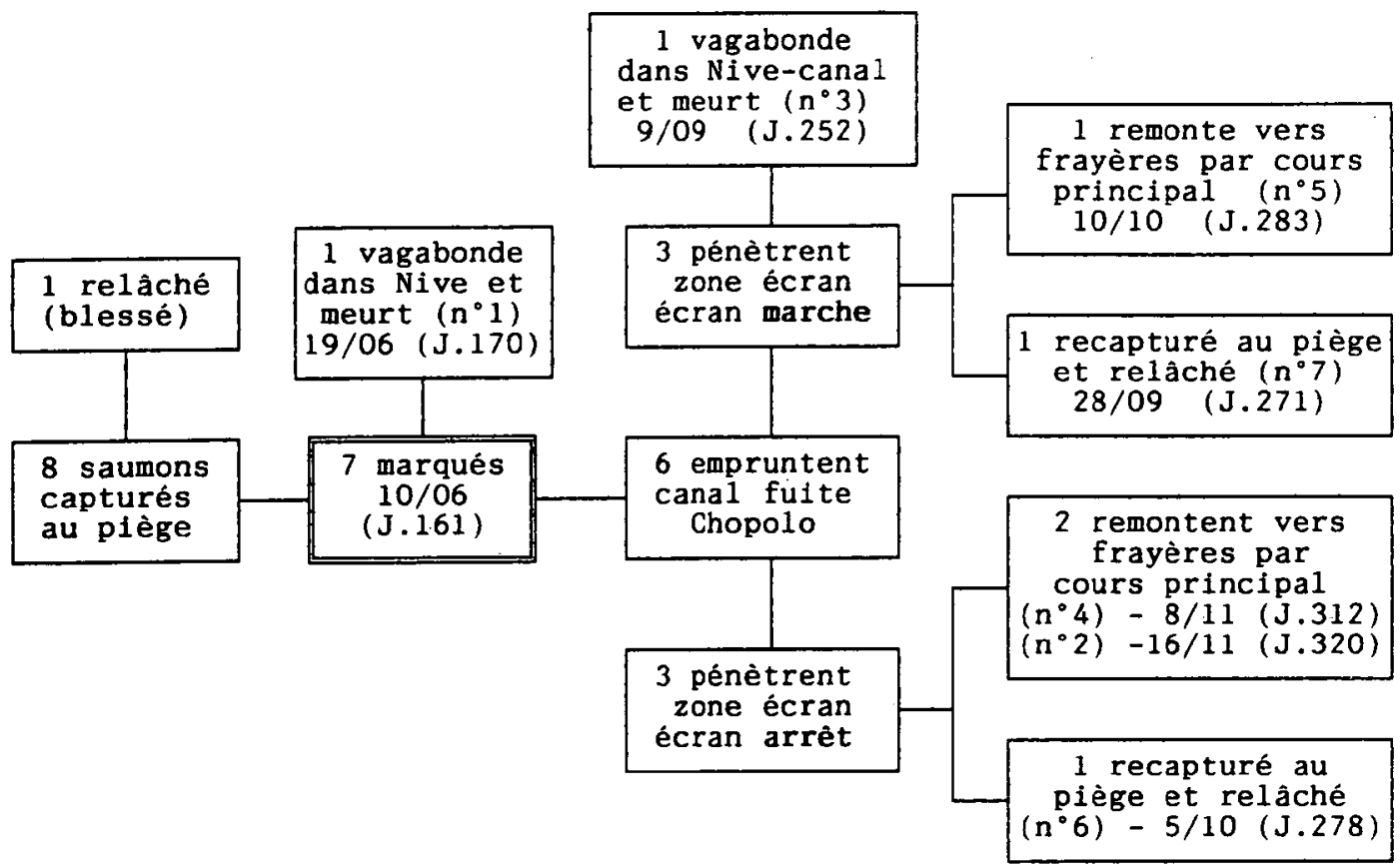

Figure 23 : Comportements migratoires schématisés des saumons dans la zone expérimentale.

Figure 23 : Diagram of the salmon migration behaviour in the experimental zone. 
- la répuision par l'écran ne semble pas perturber la prospection des diverses voies de passage vers l'amont puisque les trois poissons ont prospecté à une ou plusieurs reprises le bras court-circuité de Nive. Il est vraisemblable que les conditions défavorables de passage dans ce bras en dehors des coups d'eau (débit et vitesse très faibles) sont la raison essentielle de l'absence de passage par cette voie ;

- il ne semble pas enfin qu'il y ait "mémorisation" de longue durée de l'effet répulsif du champ électrique. En effet, l'un des poissons, repoussé à plusieurs reprises par l'écran $\left(S . n^{\circ} 7\right)$ a franchil'écran lorsqu'il a été mis hors fonctionnement sans présenter de comportement différent de celui du poisson qui n'était jamais rentré auparavant dans le champ électrique (S. $\left.n^{\circ} 6\right)$;

- les poissons ne restent pas en position d'attente à l'aval immédiat de l'écran même si celui-ci, comme c'était le cas dans la présente étude, est situé à l'amont du canal de fuite. Les trois saumons ont en effet redescendu le canal de fuite, pourtant relativement long, après répulsion par l'écran. L'un deux (S. $\left.n^{\circ} 7\right)$ est resté pendant 42 jours dans le canal de fuite, mais ce comportement a été également observé sur les autres saumons qui ont stationné pendant de longues périodes en divers points à l'aval de Chopolo. Chacun d'eux a également prospecté au moins une fois le bras court-circuité de Chopolo.

\section{DISCUSSION}

\section{Influence de la technique de pistage}

La technique de radiopistage, utilisée pour cette étude, présente des avantages et inconvénients qu'il convient de discuter avant de conclure sur les résultats obtenus. Le principal avantage est essentiellement le fait de pouvoir suivre de façon relativement précise le comportement des animaux dans le milieu. Il est donc possible d'accéder, avec un nombre restreint de poissons parfaitement individualisés, à des résultats descriptifs permettant parfois de s'affranchir du traitement statistique d'informations obtenues à partir d'un nombre élevé d'individus. Les inconvénients de la méthode sont de deux ordres : biais éventuel dû au stress du marquage et souvent difficulté de généralisation à l'ensemble d'une population du comportement d'un nombre restreint d'individus, surtout quand on constate une hétérogénéité très marquée de celui-ci.

\section{Stress de marquage}

Le stress provoqué par le marquage est très difficile à évaluer essentiellement du fait de l'individualité des comportements qui empêche la caractérisation d'individus de référence. Cependant un certain nombre de points de l'activité migratoire sont identiques et il est possible de déterminer indirectement la "normalité" du comportement des individus. Dans la présente étude, 5 poissons sur 7 ont été suivis pendant plusieurs mois au cours desquels ils ont tous manifesté une activité permanente, une réaction "normale" aux variations de conditions du milieu (débit, température...) connues pour agir sur le comportement migratoire, ainsi qu'un comportement "normal" de remontée vers les zones de frayères (Fig. 23). Le comportement de fraie de deux poissons (S. $n^{\circ} 4$ et $n^{\circ} 7$ ) a même pu être observé visuellement. Le cas des deux poissons morts respectivement 9 jours $\left(S . n^{\circ} 1\right)$ et 92 jours $\left(S . n^{\circ} 3\right)$ après marquage est plus délicat à traiter. II est impossible de savoir quelle peut être la part respective du marquage (de rares cas de mortalités ont été observés lors de précédentes opérations de radiopistage - TRAVADE et al., 1989) et celle de la mortalité naturelle (des saumons morts sont observés tous les ans dans cette zone et à cette époque par les pêcheurs - MARTY (CSP), comm. pers.). II ne semble pas en tout cas que la mortalité rapide du saumon $n^{\circ} 1$ remette en cause la prise en compte des résultats obtenus lors de la période de grande activité qu'il a manifesté avant son décès. Son comportement au voisinage de l'écran électrique est en effet apparu très semblable à celui manifesté ultérieurement par les deux autres poissons.

\section{Effectif marqué}

Compte tenu de la spécificité des comportements - on se heurte à un des inconvénients majeurs du radiopistage (cf. tête de chapitre) -, il semble inutile de rechercher à travers 
l'observation des déplacements des saumons marqués une extrapolation au comportement de l'ensemble de la population. L'observation des résultats ne permet pas non plus de dégager des tendances : activité différente en fonction de la taille par exemple (BAGLINIĖRE et al., 1991). Cette diversité observée des comportements incite à penser qu'un effectif marqué beaucoup plus important aurait été nécessaire pour une telle analyse.

\section{Influence du lieu de capture}

On peut s'interroger sur l'importance du lieu de capture des poissons sur leur comportement ultérieur. II n'a en effet pas été techniquement possible de capturer les saumons à l'aval du site et leur capture dans la passe à poissons de Chopolo peut entâcher les résultats de deux façons antagonistes : d'une part, la connaissance du site pourrait les inciter à retourner prioritairement dans le canal de fuite, d'autre part, le stress subi lors de leur capture pourraient les rendre méfiants et les dissuader de pénétrer à nouveau dans la passe. La méthodologie utilisée ne permet pas de conclure quant à l'effet de ces deux biais éventuels sur les résultats, aucun des deux n'apparaissant déterminant. Dans la mesure du possible, il est préférable d'utiliser pour de telles études des poissons "vierges", capturés le plus loin possible à l'aval du site.

\section{Influence des conditions du milieu}

II est fort probable que les faibles débits et la température élevée de l'eau soient les causes essentielles de l'arrêt migratoire des poissons à l'aval de Chopolo (cf. § 3.2.1), alors que l'écran en fonctionnement 1 jour sur 2 permettait le passage vers l'amont. Avec, en particulier, des conditions hydrauliques meilleures, il est possible que la migration se soit déroulée différemment, mais on peut cependant s'interroger sur l'influence de l'usine et de la passe dans ce processus d'arrêt estival.

Par ailleurs, aucun des poissons marqués n'est passé par le bras court-circuité en dehors des coups d'eau. Ce fait est surprenant, surtout en ce qui concerne les saumons repoussés par l'écran - les plus actifs - avant cette période d'arrêt quasi-général (saumons $n^{\circ} 1, n^{\circ} 5$ et $\left.n^{\circ} 7\right)$. On peut se demander si ceci est dû uniquement aux conditions défavorables de vitesse d'écoulement et de débit dans ce bras ou à d'autres causes qui n'ont pu être identifiées.

En tout état de cause, les conclusions concernant le comportement des saumons visà-vis de l'obstacle et dans les conditions expérimentales de cette étude restent valables.

\section{CONCLUSION}

L'étude entreprise sur la Nive à Chopolo a permis, grâce à la technique de radiopistage, d'apporter des éclaircissements sur le comportement in situ de saumons devant un écran électrique répulsif. L'effet du champ électrique ne produit pas de perturbations profondes du comportement des poissons, qui poursuivent leurs déplacements dans la rivière sans dévaler de façon excessive ni irréversible. II ne semble pas y avoir non plus de bloquage de longue durée des poissons à l'aval immédiat de l'écran : ils ont en effet effectué d'autres prospections dans la rivière après avoir redescendu le long canal de fuite à l'extrémité duquel était implanté l'écran. II ne semble pas enfin qu'il y ait mémorisation de longue durée du blocage par l'écran : après avoir été repoussés à plusieurs reprises par l'écran en marche, les poissons se sont montrés capables de le franchir après sa mise hors service.

Les poissons repoussés par l'écran n'ont pas poursuivi leur migration par le bras courtcircuité en dehors des coups d'eau. Cela peut avoir plusieurs causes qui n'ont pu être identifiées : inadéquation du débit et de la vitesse de l'eau dans ce bras, mémorisation par le poisson de l'attractivité supérieure du canal de fuite accentuée par le fait que l'écran était disposé à son extrémité amont, nécessité d'un stimulus migratoire fort pourvaincre les difficultés de passage d'un obstacle. Ces points importants dans la mise en œuvre pratique d'un écran électrique comme alternative à la construction d'un ouvrage de franchissement mériteraient d'être approfondis. A ce titre, il serait intéressant de pouvoir tester l'efficacité de cet écran situé au confluent du canal de fuite et de la rivière. 


\section{REMERCIEMENTS}

Cette étude a pu être réalisée grâce à la collaboration INRA (Station d'Hydrobiologie de St-Pée-sur-Nivelle) et EDF (Direction des Etudes et Recherches).

Nous tenons à remercier :

- le Conseil Régional Midi-Pyrénées pour l'aide financière qui a permis la construction de l'écran électrique ;

- le propriétaire de la microcentrale de Chopolo, Monsieur Duhalde, pour sa compréhension et son aide matérielle ;

- le Conseil Supérieur de la Pêche (D.R. Toulouse, Antenne de Pau) pour son aide matérielle au cours de l'expérimentation ;

- l'Association Agréée de Pêche et Pisciculture de la Nive pour avoir autorisé cette étude et contribué au bon déroulement de l'expérimentation.

\section{BIBLIOGRAPHIE}

BAGLINIÈRE J.L., MAISSE G., NIHOUARN A., 1991. Radiotracking of male adult Atlantic salmon, Salmo salar L., during the last phase of spawning migration in a spawning stream (Brittany, France). Aquat. Living Resour., 4, 161-167.

BARIL D., GUENEAU P., 1986. Radiopistage de saumon adulte (Salmo salar) en Loire. Bull. Fr. Pêche Piscic., 302, 86-105.

BOMASSIP., BRUGELC., 1991. Etude de la migration des saumons sur le Haut-Allier. Problèmes posés par l'aménagement hydro-électrique de Poutès-Monistrol. Rapp. CSP, 64 p. + 59 p. tableaux et figures.

BURGER C., HEPLER K., ROTH K., DELANEY K., 1981. Radiotracking of adult coho salmon (Oncorhynchus kisutch) in the Deshka River Alaska. Proc. third int. conf. on wildlife biotelemetry, Laramie, Wyoming, 151-158.

GOSSET C., 1989. Etude sur l'installation d'écrans électriques à poissons. Convention d'étude Région Midi-Pyrénées-INRA. Rapp. tech. INRA, St-Pée-sur-Nivelle, $55 \mathrm{p}$.

HARTLEY W.G., SIMPSON D., 1968. Ecrans électriques à poissons au Royaume-Uni. In : Vibert R., Applications de l'électricité à la biologie et à l'aménagement des pêches continentales, 191-206. Symposium CECPI, Belgrade, 1966, INRA Publ., Paris.

JOHNSON I.K., BEAUMONT W.R.C., WELTON J.S., 1990. The use of electric fish screens in the Hampshire Test and Itchem, England. In Cowx I.G., Development in electric fishing. Proceedings of an International Symposium on Fishing with Electricity, Hull, UK, 1988. Fishing News Books, Blackwell Scientific Publications Ltd, Oxford.

MC LEAVE J.D., POWER J.H., ROMMEL S.A., 1978. Use of radio telemetry for studying upriver migration of adult Atlantic Salmon (Salmo salar). J. Fish Biol., 12, 549-558.

SOLOMON D.J., 1982. Tracking fish with radiotags. Symp. Zool. Soc. Lond., 49, 95-105.

STEINBACH P., GUENEAU P., AUTUORO A., BROUSSARD D., 1986. Radiopistage de la grande alose en Loire. Bull. Fr. Pêche Piscic., 302, 106-117.

STEWART P.A.M., 1990. Electric screens and guides. In Cowx I.G. and Lamarque P., Fishing with electricity, 140-156. Fishing News Books, Blackwell Scientific Publications Ltd, Oxford.

STRAKKOV V.A., 1965. Electricesky rybozagraditel tipa ERZU-1, evo restrjstvo, vybor i rasect parametrov. Trudy koorinationnych sovescanij po Gidrotechnike, 24, 67-70. Tra. Fr. 1988, INRA, St-Pée-sur-Nivelle.

TRAVADE F., BOMASSI J.M., BACH J.M., BRUGEL C., STEINBACH P., LUQUET J.F., PUSTELNIK G., 1989. Use of radiotracking in France for recent studies concerning the EDF fishways program. Hydro-écol. Appl., 33-51. 